\title{
DIRECTIONALLY CONVEX ORDERING OF RANDOM MEASURES, SHOT NOISE FIELDS, AND SOME APPLICATIONS TO WIRELESS COMMUNICATIONS
}

\author{
BARTŁOMIEJ BŁASZCZYSZYN,* INRIA-ENS and University of Wroctaw \\ D. YOGESHWARAN, ${ }^{*} * *$ INRIA-ENS
}

\begin{abstract}
Directionally convex ordering is a useful tool for comparing the dependence structure of random vectors, which also takes into account the variability of the marginal distributions. It can be extended to random fields by comparing all finite-dimensional distributions. Viewing locally finite measures as nonnegative fields of measure values indexed by the bounded Borel subsets of the space, in this paper we formulate and study directionally convex ordering of random measures on locally compact spaces. We show that the directionally convex order is preserved under some of the natural operations considered on random measures and point processes, such as deterministic displacement of points, independent superposition, and thinning, as well as independent, identically distributed marking. Further operations on Cox point processes such as position-dependent marking and displacement of points are shown to preserve the order. We also examine the impact of the directionally convex order on the second moment properties, in particular on clustering and on Palm distributions. Comparisons of Ripley's functions and pair correlation functions, as well as examples, seem to indicate that point processes higher in the directionally convex order cluster more. In our main result we show that nonnegative integral shot noise fields with respect to the directionally convex ordered random measures inherit this ordering from the measures. Numerous applications of this result are shown, in particular to comparison of various Cox processes and some performance measures of wireless networks, in both of which shot noise fields appear as key ingredients. We also mention a few pertinent open questions.
\end{abstract}

Keywords: Stochastic ordering; directional convexity; random measure; random field; point process; wireless network

2000 Mathematics Subject Classification: Primary 60E15

Secondary 60G60; 60G57; 60G55

\section{Introduction}

Point processes (PPs) have been at the center of various studies in stochastic geometry, both theoretical and applied. Most of the work involving the quantitative analysis of PPs has dealt with Poisson PPs. One of the main reasons being that the characteristics of Poisson PPs are amenable to computations and yield nice closed-form expressions in many cases. Computations have been difficult in a great number of cases, even for Cox (doubly stochastic Poisson) PPs.

Received 12 June 2008; revision received 2 April 2009.

* Postal address: Bureau 21 TREC, INRIA (5th floor), 23 Avenue d'Italie, CS 81321, 75214 Paris Cedex 13, France.

** Email address: yogesh@di.ens.fr 


\subsection{Comparison of point processes}

To improve upon this situation, qualitative, comparative studies of PPs have emerged as useful tools. The first method of comparison of PPs has been coupling or stochastic domination (see [17], [19], and [31]). In our terminology, these are known as the strong ordering of PPs. When two PPs can be coupled, one turns out to be a subset of the other. This ordering is very useful for obtaining various bounds and proving limit theorems. However, using it means that we cannot compare two different PPs with the same mean measure. An obvious example is a homogeneous Poisson PP and a stationary Cox PP with the same intensity. The question arises of what ordering is suitable for such PPs. This is an important question since it is expected that by comparing PPs of the same intensity we should be able to achieve a tighter bound than by coupling. For some more details on the strong ordering of PPs and the need for other orders, see the remarks in [28, Sections 5.4 and 7.4.2].

\subsection{From the convex to the directionally convex order}

Two random variables $X$ and $Y$ with the same mean $\mathrm{E}(X)=\mathrm{E}(Y)$ can be compared by how 'spread out' their distributions are. This statistical variability (in a statistical ensemble) is captured to a limited extent by the variance, but more fully by convex ordering, under which $X$ is less than $Y$ if and only if, for all convex $f, \mathrm{E}(f(A)) \leq \mathrm{E}(f(B))$. In multidimension, besides different statistical variability of marginal distributions, two random vectors can exhibit different dependence properties on their coordinates. The most evident example here is the comparison between a vector composed of several copies of one random variable and a vector composed of independent copies sampled from the same distribution. A useful tool for comparing the dependence structure of random vectors with fixed marginals is the supermodular order. The directionally convex order is another integral order (generated by a class of directionally convex functions in the same manner as convex functions generate the convex order) that can be seen as a generalization of the supermodular order, which in addition takes into account the variability of the marginals (cf. [28, Section 3.12]). It can be naturally extended to random fields by comparing all finite-dimensional distributions.

\subsection{The directionally convex order of random measures}

In this paper we make the further obvious extension of viewing the directionally convex ordering of locally finite measures (to which PPs belong) as nonnegative fields of measure values on all bounded subsets of the space. We show that the directionally convex order is preserved under some of the natural operations considered on random measures and PPs, such as independent superposition and thinning. Also, we examine the impact of the directionally convex order on the second moment properties, in particular on clustering and Palm distributions.

\subsection{Integral shot noise fields}

Many interesting characteristics of random measures, in both theory and applications, have the form of integrals of some nonnegative kernels. We call them integral shot noise fields. For example, many classes of Cox PPs, with the most general being Lévy-based Cox PPs (cf. [12]), have stochastic intensity fields, which are shot noise fields. They are also key ingredients of the recently proposed, so-called 'physical' models for wireless networks, as we shall explain in what follows (see also [1], [6], and [9]). It is therefore particularly appealing to study the shot noise fields generated by directionally convex-ordered random measures.

Since integrals are linear operators on the space of measures, and knowing that a linear function of a vector is trivially directionally convex, it is natural to expect that the integral 
shot noise fields with respect to directionally convex-ordered random measures will inherit this ordering from the measures. However, this property cannot be concluded immediately from the finite-dimensional directionally convex ordering of measures. The formal proof of this fact, which is the main result of this paper, involves some arguments from the theory of integration combined with the closure property of the directionally convex order under joint weak convergence and convergence in mean.

\subsection{Ordering in queueing theory and wireless communications}

The theory of stochastic ordering provides elegant and efficient tools for the comparison of random objects and is now being used in many fields. In particular, in queueing theory context, Ross [32] conjectured that replacing a stationary Poisson arrival process in a single-server queue by a stationary Cox PP with the same intensity should increase the average customer delay. There have been many variations of this conjecture, which are now known as Ross-type conjectures. They triggered interest in the comparison of queues with similar inputs (see [4], [24], and [30]). The notion of a directionally convex function was partially developed and used in conjunction with the proofs of Ross-type conjectures (see [20], [21], and [33]). Much earlier to these works, a comparative study of queues motivated by neuron-firing models can be found in [14] and [15]. Also, the comparison of variances of PPs and fibre processes was studied in [34] and, hence, [34] can be considered as a precursor to our paper. The applicability of these results has generated interest in the theory of stochastic ordering, as can be seen from the diverse results in the book of Müller and Stoyan [28]. As most works on the ordering of PPs were motivated by applications to queueing theory, results were primarily focused on one-dimensional PPs. An attempt to rectify the lack of work in higher dimensions was made in [23], where comparison results for shot noise fields of spatial stationary Cox PPs were given. The results of [23] are the starting point of our investigation.

Our interest in the ordering of PPs, and in particular in the shot noise fields they generate, has roots in the analysis of wireless communications, where these objects are primarily used to model the so-called interference, that is, the total power received from many emitters scattered in the plane or space and sharing the common Hertzian medium. According to a new emerging methodology, the interference-aware stochastic geometry modeling of wireless communications provides a way of defining and computing macroscopic properties of large wireless networks by some averaging over all potential random patterns for node locations in an infinite plane and radio channel characteristics, in the same way as queueing theory provides average response times or congestion over all potential arrival patterns within a given parametric class. These macroscopic properties allow one to characterize the key dependencies of the network performance characteristics in terms of a relatively small number of parameters.

In the above context, the Poisson distribution of emitters/receivers/users is often too simplistic. Statistics show that the real patterns of users exhibit more clustering effects ('hotspots') than observed in a homogeneous Poisson PP. On the other hand, a good packet-collisionavoidance mechanism scheme should create some 'repulsion' in the pattern of nodes allowed to access simultaneously the channel. This raises questions about the analysis of non-Poisson models, which could to some extent be tackled on the grounds of stochastic ordering theory. Interestingly, we shall show that there are certain performance characteristics in wireless networks that improve with more variability in the input process.

\subsection{Outline of the paper}

In Section 2 we present the main definitions and state the main results concerning directionally convex ordering of the integral shot noise fields. In Section 3 we explore the various 
consequences of ordering of random measures. The proofs of the main results are given in Section 4. Examples illustrating the use and application of the theorems will be presented in Section 5. In Section 6 we sketch some of the possible applications of the results in the context of wireless communications. Finally, we conclude with some remarks and questions in Section 7. Appendix A contains some properties and extensions of stochastic orders that are used in the paper.

\section{Definitions and the main result}

The order ' $\leq$ ' on $\mathbb{R}^{n}$ shall denote the componentwise partial order, i.e. $\left(x_{1}, \ldots, x_{n}\right) \leq$ $\left(y_{1}, \ldots, y_{n}\right)$ if $x_{i} \leq y_{i}$ for every $i$.

Definition 2.1. 1. We say that a function $f: \mathbb{R}^{d} \rightarrow R$ is directionally convex (dcx) if, for every $x, y, p, q \in \mathbb{R}^{d}$ such that $p \leq x, y \leq q$, and $x+y=p+q$,

$$
f(x)+f(y) \leq f(p)+f(q) .
$$

2. A function $f$ is said to be directionally concave ( $\mathrm{dcv}$ ) if the inequality in the above equation is reversed.

3. A function $f$ is said to be directionally linear $(\mathrm{dl})$ if it is $\mathrm{dcx}$ and $\mathrm{dcv}$.

A function $f=\left(f_{1}, \ldots, f_{n}\right): \mathbb{R}^{d} \rightarrow \mathbb{R}^{n}$ is said to be dcx or dcv if each of its component $f_{i}$ is dex or, respectively, dcv. Also, we shall abbreviate increasing and dex by idcx and decreasing and dcx by ddcx. Similar abbreviations shall be used for dcv and dl functions.

In the following, let $\mathfrak{F}$ denote some class of functions from $\mathbb{R}^{d}$ to $\mathbb{R}$. The dimension $d$ is assumed to be clear from the context. Unless mentioned otherwise, when we state $\mathrm{E}(f(X))$ for $f \in \mathfrak{F}$ and a random vector $X$, we assume that the expectation exists, i.e. for each random vector $X$, we consider the subclass of $\mathfrak{F}$ for which the expectations exist with respect to $X$.

Definition 2.2. 1. Suppose that $X$ and $Y$ are real-valued random vectors of the same dimension. Then $X$ is said to be less than $Y$ in the $\mathfrak{F}$ order if $\mathrm{E}(f(X)) \leq \mathrm{E}(f(Y))$ for all $f \in \mathfrak{F}$ (for which both expectations are finite). We shall denote this order by $X \leq \mathfrak{F} Y$.

2. Suppose that $\{X(s)\}_{s \in S}$ and $\{Y(s)\}_{s \in S}$ are real-valued random fields, where $S$ is an arbitrary index set. We say that $\{X(s)\} \leq_{\mathfrak{F}}\{Y(s)\}$ if, for every $n \geq 1$ and $s_{1}, \ldots, s_{n} \in S$, $\left(X\left(s_{1}\right), \ldots, X\left(s_{n}\right)\right) \leq \mathfrak{F}\left(Y\left(s_{1}\right), \ldots, Y\left(s_{n}\right)\right)$.

In the remaining part of the paper, we mainly consider $\mathfrak{F}$ to be the class of dcx, idcx, and idcv functions; the negation of these functions gives rise to the dcv, ddcv, and ddcx orders, respectively. If $\mathfrak{F}$ is the class of increasing functions, we shall replace $\mathfrak{F}$ by st (strong) in the above definitions. Such notation is standardly used in the literature.

As concerns random measures, we shall work in the setup of [16]. Let $\mathbb{E}$ be a locally compact, second countable (LCSC) Hausdorff space. Such spaces are polish, i.e. a complete and separable metric space. Let $\mathrm{B}(\mathbb{E})$ be the Borel $\sigma$-algebra, and let $\mathrm{B}_{\mathrm{b}}(\mathbb{E})$ be the $\sigma$-ring of bounded Borel subsets. Let $\mathbb{M}=\mathbb{M}(\mathbb{E})$ be the space of nonnegative Radon measures on $\mathbb{E}$. The Borel $\sigma$-algebra $\mathcal{M}$ is generated by the mappings $\mu \mapsto \mu(B)$ for all bounded Borel subsets $B$. A random measure $\Lambda$ is a mapping from a probability space $(\Omega, \mathcal{F}, \mathrm{P})$ to $(\mathbb{M}, \mathcal{M})$. We shall call a random measure $\Phi$ a PP if $\Phi \in \overline{\mathbb{N}}$, the subset of counting measures in $\mathbb{M}$. Furthermore, we shall say that a PP $\Phi$ is simple if, almost surely, $\Phi(\{x\}) \leq 1$ for all $x \in \mathbb{E}$. Throughout, we shall use $\Lambda$ for an arbitrary random measure and $\Phi$ for a PP. A random measure $\Lambda$ can be 


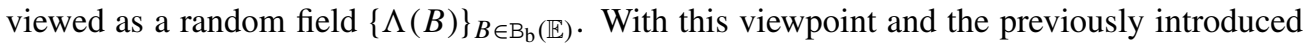
notion of ordering of random fields, we define ordering of random measures.

Definition 2.3. Suppose that $\Lambda_{1}(\cdot)$ and $\Lambda_{2}(\cdot)$ are random measures on $\mathbb{E}$. Then we say that $\Lambda_{1}(\cdot) \leq_{\mathrm{dcx}} \Lambda_{2}(\cdot)$ if, for any bounded Borel subsets $I_{1}, \ldots, I_{n}$ in $\mathbb{E}$,

$$
\left(\Lambda_{1}\left(I_{1}\right), \ldots, \Lambda_{1}\left(I_{n}\right)\right) \leq_{\mathrm{dcx}}\left(\Lambda_{2}\left(I_{1}\right), \ldots, \Lambda_{2}\left(I_{n}\right)\right) .
$$

The definition is similar for the other orders, i.e. when $\mathfrak{F}$ is the class of idcx, idcv, ddcx, ddcv, or st functions.

Definition 2.4. Let $S$ be any set, and let $\mathbb{E}$ be an LCSC space. Given a random measure $\Lambda$ on $\mathbb{E}$ and a measurable (in the first variable alone) response function $h(x, y): \mathbb{E} \times S \rightarrow \overline{\mathbb{R}}^{+}$, where $\overline{\mathbb{R}}^{+}$denotes the completion of the positive real line with $\infty$, the (integral) shot noise field is defined as

$$
V_{\Lambda}(y)=\int_{\mathbb{E}} h(x, y) \Lambda(\mathrm{d} x) .
$$

With this brief introduction, we are ready to state our key result, which will be proved in Section 4.1.

Theorem 2.1. 1. If $\Lambda_{1} \leq_{\text {idcx }} \Lambda_{2}$ then $\left\{V_{\Lambda_{1}}(y)\right\}_{y \in S} \leq_{\text {idcx }}\left\{V_{\Lambda_{2}}(y)\right\}_{y \in S}$, and if $\Lambda_{1} \leq_{\text {idcv }} \Lambda_{2}$ then $\left\{V_{\Lambda_{1}}(y)\right\}_{y \in S} \leq$ idcv .

2. Let $\mathrm{E}\left(V_{\Lambda_{i}}(y)\right)<\infty$ for all $y \in S, i=1$, 2. If $\Lambda_{1} \leq_{\mathrm{dcx}} \Lambda_{2}$ then $\left\{V_{\Lambda_{1}}(y)\right\}_{y \in S} \leq_{\mathrm{dcx}}$ $\left\{V_{\Lambda_{2}}(y)\right\}_{y \in S}$.

The first part of the above theorem for the one-dimensional marginals of bounded shot noise fields generated by lower semicontinuous response functions is proved in [23] for the special case of spatial stationary Cox PPs. It is conspicuous that we have generalized the earlier result to a great extent. This more general result will be used in many places in this paper, in particular to prove ordering of independently, identically marked PPs (Proposition 3.2), Ripley's functions (Proposition 3.4), Palm measures (Proposition 3.5), independently marked Cox processes (Proposition 3.7), and extremal shot noise fields (Proposition 4.1). Apart from these results, in Sections 5 and 6 we shall provide examples and applications that will amply demonstrate the use of Theorem 2.1 .

\section{Ordering of random measures and point processes}

We shall now give a sufficient condition for random measures to be ordered, namely that condition (2.1) in Definition 2.3 needs to be verified only for disjoint, bounded Borel subsets. The necessity is trivial. This is a much easier condition and will be used many times in the remaining part of the paper.

Proposition 3.1. Suppose that $\Lambda_{1}(\cdot)$ and $\Lambda_{2}(\cdot)$ are two random measures on $\mathbb{E}$. Then $\Lambda_{1}(\cdot) \leq_{\mathrm{dcx}} \Lambda_{2}(\cdot)$ if and only if condition (2.1) holds for all mutually disjoint, bounded Borel subsets. The same result holds true for the idcx and idcv orders.

Proof. We only need to prove the 'if' part. We shall prove the result for the dcx order; the same argument is valid for $f$ being idcx or idcv. Let condition (2.1) be satisfied for all mutually disjoint, bounded Borel subsets. Let $f: \mathbb{R}_{+}^{n} \rightarrow R$ be a dcx function, and let $B_{1}, \ldots, B_{n}$ be bounded Borel subsets. We can choose mutually disjoint, bounded Borel subsets $A_{1}, \ldots, A_{m}$ such that $B_{i}=\bigcup_{j \in J_{i}} A_{j}$ for all $i$. Hence, $\Lambda\left(B_{i}\right)=\sum_{j \in J_{i}} \Lambda\left(A_{j}\right)$. Now define $g: \mathbb{R}_{+}^{m} \rightarrow \mathbb{R}_{+}^{n}$ 
as $g\left(x_{1}, \ldots, x_{m}\right)=\left(\sum_{j \in J_{1}} x_{j}, \ldots, \sum_{j \in J_{n}} x_{j}\right)$. Then $g$ is idl and so $f \circ g$ is dex. Moreover, $f\left(\Lambda\left(B_{1}\right), \ldots, \Lambda\left(B_{n}\right)\right)=f \circ g\left(\Lambda\left(A_{1}\right), \ldots, \Lambda\left(A_{m}\right)\right)$ and, thus, the result for dex follows.

\subsection{Simple operations preserving order}

PPs are special cases of random measures and as such will be subject to the considered ordering. It is known that each PP $\Phi$ on an LCSC space $\mathbb{E}$ can be represented as a countable sum $\Phi=\sum_{i} \varepsilon_{X_{i}}$ of Dirac measures $\left(\varepsilon_{x}(A)=1\right.$ if $x \in A$ and 0 otherwise) in such a way that $X_{i}$ are random elements in $\mathbb{E}$. We shall now show that all the three orders, dcx, idcx, and idcv, preserve some simple operations on random measures and PPs, such as deterministic mapping, independent, identically distributed (i.i.d.) thinning, and independent superposition.

Let $\phi: \mathbb{E} \rightarrow \mathbb{E}^{\prime}$ be a measurable mapping to some LCSC space $\mathbb{E}^{\prime}$. By the image of a (random) measure $\Lambda$ by $\phi$ we mean $\Lambda^{\prime}(\cdot)=\Lambda\left(\phi^{-1}(\cdot)\right)$. Note that the image of a PP $\Phi$ by $\phi$ consists of a deterministic displacement of all its points by $\phi$.

Let $\Phi=\sum_{i} \varepsilon_{x_{i}}$. By i.i.d. marking of $\Phi$, with marks in some LCSC space $\mathbb{E}^{\prime}$, we mean a PP on the product space $\mathbb{E} \times \mathbb{E}^{\prime}$, with the usual product Borel $\sigma$-algebra, defined by $\tilde{\Phi}=\sum_{i} \varepsilon_{\left(x_{i}, Z_{i}\right)}$, where $\left\{Z_{i}\right\}$ are i.i.d. random variables (RVs), so-called marks, on $\mathbb{E}^{\prime}$. By i.i.d. thinning of $\Phi$, we mean $\bar{\Phi}=\sum_{i} Z_{i} \varepsilon_{x_{i}}$, where $Z_{i}$ are i.i.d. $0-1$ Bernoulli RVs. The probability $\mathrm{P}(Z=1)$ is called the retention probability. Superposition of PPs is understood as the addition of (counting) measures. Measures on Cartesian products of LCSC spaces are always considered with their corresponding product Borel $\sigma$-algebras.

Proposition 3.2. Suppose that $\Lambda_{i}, i=1,2$, are random measures and that $\Phi_{i}, i=1,2$, are PPs. Assume that $\Lambda_{1} \leq_{\mathrm{dcx}} \Lambda_{2}, \Lambda_{1} \leq_{\mathrm{idcx}} \Lambda_{2}, \Lambda_{1} \leq_{\mathrm{idcv}} \Lambda_{2}$, and $\Phi_{1} \leq_{\mathrm{dcx}} \Phi_{2}, \Phi_{1} \leq_{\mathrm{idcx}} \Phi_{2}$, $\Phi_{1} \leq_{\text {idcv }} \Phi_{2}$.

1. Let $\Lambda_{i}^{\prime}$ be the image of $\Lambda_{i}, i=1,2$, by some mapping $\phi: \mathbb{E} \rightarrow \mathbb{E}^{\prime}$. Then $\Lambda_{1}^{\prime} \leq_{\mathrm{dcx}} \Lambda_{2}^{\prime}$, $\Lambda_{1}^{\prime} \leq_{\mathrm{idcx}} \Lambda_{2}^{\prime}$, and $\Lambda_{1}^{\prime} \leq_{\mathrm{idcv}} \Lambda_{2}^{\prime}$. As a special case, the same holds true for the displacement of the $\Phi_{i}$ points by $\phi$.

2. Let the $\Phi_{i}, i=1,2$, be simple PPs and let the $\tilde{\Phi}_{i}, i=1,2$, be the corresponding i.i.d. marked PPs with the same distribution of marks. Then $\tilde{\Phi}_{1} \leq_{\mathrm{dcx}} \tilde{\Phi}_{2}, \tilde{\Phi}_{1} \leq_{\mathrm{idcx}} \tilde{\Phi}_{2}$, and $\tilde{\Phi}_{1} \leq_{\text {idev }} \tilde{\Phi}_{2}$.

3. Let $\bar{\Phi}_{i}$ be the i.i.d. thinning of $\Phi_{i}, i=1,2$, with the same retention probability. Then $\bar{\Phi}_{1} \leq_{\mathrm{dcx}} \bar{\Phi}_{2}, \bar{\Phi}_{1} \leq_{\mathrm{idcx}} \bar{\Phi}_{2}$, and $\bar{\Phi}_{1} \leq_{\mathrm{idcv}} \bar{\Phi}_{2}$.

4. Let $\Lambda_{1}^{\prime}$ and $\Lambda_{2}^{\prime}$ be two random measures such that $\Lambda_{1}^{\prime} \leq_{\mathrm{dcx}} \Lambda_{2}^{\prime}, \Lambda_{1}^{\prime} \leq_{\mathrm{idcx}} \Lambda_{2}^{\prime}$, and $\Lambda_{1}^{\prime} \leq_{\mathrm{idcv}} \Lambda_{2}^{\prime}$. Assume that the $\Lambda_{i}^{\prime}$ s are independent of the $\Lambda_{i}$ s. Then $\Lambda_{1}+\Lambda_{1}^{\prime} \leq_{\mathrm{dcx}}$ $\Lambda_{2}+\Lambda_{2}^{\prime}, \Lambda_{1}+\Lambda_{1}^{\prime} \leq_{\mathrm{idcx}} \Lambda_{2}+\Lambda_{2}^{\prime}$, and $\Lambda_{1}+\Lambda_{1}^{\prime} \leq_{\mathrm{idcv}} \Lambda_{2}+\Lambda_{2}^{\prime}$, where '+' is understood as the addition of measures.

5. Suppose that the random measures are on the product space $\mathbb{E} \times \mathbb{E}^{\prime}$. Then $\Lambda_{1}(\mathbb{E} \times \cdot) \leq_{\mathrm{dcx}}$ $\Lambda_{2}(\mathbb{E} \times \cdot), \Lambda_{1}(\mathbb{E} \times \cdot) \leq$ idcx $\Lambda_{2}(\mathbb{E} \times \cdot)$, and $\Lambda_{1}(\mathbb{E} \times \cdot) \leq$ idcv $\Lambda_{2}(\mathbb{E} \times \cdot)$, provided that the respective projections are Radon measures.

Proof. 1. The result follows immediately from Definition 2.3.

2. We shall prove that $\tilde{\Phi}_{1} \leq_{\mathrm{dcx}} \tilde{\Phi}_{2}$; the proof for the other orders is similar. Since $\mathbb{E}$ is an LCSC space, there exists a null array of partitions $\left\{B_{n, j} \subset \mathbb{E}\right\}_{n \geq 1, j \geq 1}$, i.e. the $\left\{B_{n, j}\right\}_{j \geq 1}$ form a finite partition of $\mathbb{E}$ for every $n$ and $\max _{j \geq 1}\left\{\left|B_{n, j}\right|\right\} \rightarrow 0$ as $n \rightarrow \infty$, where $|\cdot|$ denotes the diameter in any fixed metric (see [16, p. 11]). For every $x \in \mathbb{E}$, let $j(n, x)$ be the unique index such 
that $x \in B_{n, j(n, x)}$. Let $\bar{Z}=\left\{Z_{n, j}\right\}_{n \geq 1, j \geq 1}$ be a family of $\mathbb{E}^{\prime}$-valued i.i.d. RVs with distribution $F(\cdot)$. Define marked PPs by $\tilde{\Phi}_{i}^{n}=\sum_{X_{k} \in \Phi_{i}} \varepsilon_{\left(X_{k}, Z_{n, j\left(n, X_{k}\right)}\right)}$ for $i=1,2$. We shall now verify that the $\tilde{\Phi}_{i}^{n}$ sequences satisfy the assumption of Lemma A.2 with limits $\tilde{\Phi}_{i}$ s, respectively.

Firstly, let $B_{1}, \ldots, B_{m} \subset \mathbb{E} \times \mathbb{E}^{\prime}$ be bounded Borel subsets and let $g: \mathbb{R}^{m} \rightarrow \mathbb{R}$ be a continuous bounded function. Since the $B_{i} \mathrm{~s}$ are bounded and the $\Phi_{i} \mathrm{~S}$ are simple, given $\Phi_{i}, i=1,2$, there almost surely (a.s.) exists an $N\left(\Phi_{i}\right) \in \mathbb{N}$ such that, for $n \geq N\left(\Phi_{i}\right)$, the indices $j\left(n, X_{k}\right) \neq j\left(n, X_{l}\right)$ for $X_{k} \neq X_{l}, X_{k}, X_{l} \in \Phi_{i} \cap\left(B_{1} \cup \cdots \cup B_{m}\right)$. Hence, for $n \geq N\left(\Phi_{i}\right), \mathrm{E}\left(g\left(\tilde{\Phi}_{i}^{n}\left(B_{1}\right), \ldots, \tilde{\Phi}_{i}^{n}\left(B_{m}\right)\right) \mid \Phi_{i}\right)=\mathrm{E}\left(g\left(\tilde{\Phi}_{i}\left(B_{1}\right), \ldots, \tilde{\Phi}_{i}\left(B_{m}\right)\right) \mid \Phi_{i}\right)$ and, consequently, $\mathrm{E}\left(g\left(\tilde{\Phi}_{i}^{n}\left(B_{1}\right), \ldots, \tilde{\Phi}_{i}^{n}\left(B_{m}\right)\right) \mid \Phi_{i}\right) \rightarrow \mathrm{E}\left(g\left(\tilde{\Phi}_{i}\left(B_{1}\right), \ldots, \tilde{\Phi}_{i}\left(B_{m}\right)\right) \mid \Phi_{i}\right)$ a.s. as $n \rightarrow \infty$. Since $g$ is bounded, by the dominated convergence theorem we have

$$
\mathrm{E}\left(g\left(\tilde{\Phi}_{i}^{n}\left(B_{1}\right), \ldots, \tilde{\Phi}_{i}^{n}\left(B_{m}\right)\right)\right) \rightarrow \mathrm{E}\left(g\left(\tilde{\Phi}_{i}\left(B_{1}\right), \ldots, \tilde{\Phi}_{i}\left(B_{m}\right)\right)\right) .
$$

Thus, $\left(\tilde{\Phi}_{i}^{n}\left(B_{1}\right), \ldots, \tilde{\Phi}_{i}^{n}\left(B_{m}\right)\right) \stackrel{\mathrm{D}}{\rightarrow}\left(\tilde{\Phi}_{i}^{n}\left(B_{1}\right), \ldots, \tilde{\Phi}_{i}^{n}\left(B_{m}\right)\right)$, where $\stackrel{\text { D }}{\rightarrow}$ ' denotes convergence in distribution. Secondly, it is easy to check that, for $B_{1}=B^{\prime} \times B^{\prime \prime}$, we have $\mathrm{E}\left(\tilde{\Phi}_{i}^{n}\left(B_{1}\right)\right)=$ $\mathrm{E}\left(\Phi_{i}\left(B^{\prime}\right)\right) F\left(B^{\prime \prime}\right)=\mathrm{E}\left(\tilde{\Phi}_{i}\left(B_{1}\right)\right)$ and, hence, by an appropriate approximation, $\mathrm{E}\left(\tilde{\Phi}_{i}^{n}\left(B_{1}\right)\right)=$ $\mathrm{E}\left(\tilde{\Phi}_{i}\left(B_{1}\right)\right)$ for any bounded Borel subset $B_{1}$.

Finally, for any bounded Borel subset $B \subset \mathbb{E} \times \mathbb{E}^{\prime}$ and any realization $\bar{Z}=\bar{z}=\left\{z_{n, j}\right\}_{n \geq 1, j \geq 1}$, define $V_{i}^{\bar{z}}(B):=\int_{\mathbb{E}} \mathbf{1}\left[\left(x, z_{n, j(n, x)}\right) \in B\right] \Phi_{i}(\mathrm{~d} x)$. Since $z_{n, j(n, \cdot)}$ is a piecewise constant function, $\mathbf{1}\left[\left(x, z_{n, j(n, x)}\right) \in B\right]$ is a measurable function in $x$ and so the $V_{i}^{\bar{z}}$ s are integral shot noise fields (as per Definition 2.4) indexed by bounded Borel subsets of $\mathbb{E} \times \mathbb{E}^{\prime}$. Thus, from Theorem 2.1 we have, for any dex function $f$,

$$
\begin{aligned}
\mathrm{E}\left(f\left(\tilde{\Phi}_{1}^{n}\left(B_{1}\right), \ldots, \tilde{\Phi}_{1}^{n}\left(B_{m}\right)\right) \mid \bar{Z}=\bar{z}\right) & =\mathrm{E}\left(f\left(V_{1}^{\bar{z}}\left(B_{1}\right), \ldots, V_{1}^{\bar{z}}\left(B_{m}\right)\right)\right) \\
& \leq \mathrm{E}\left(f\left(V_{2}^{\bar{z}}\left(B_{1}\right), \ldots, V_{2}^{\bar{z}}\left(B_{m}\right)\right)\right) \\
& =\mathrm{E}\left(f\left(\tilde{\Phi}_{2}^{n}\left(B_{1}\right), \ldots, \tilde{\Phi}_{2}^{n}\left(B_{m}\right)\right) \mid \bar{Z}=\bar{z}\right) .
\end{aligned}
$$

Now, taking further expectations we obtain

$$
\left(\tilde{\Phi}_{1}^{n}\left(B_{1}\right), \ldots, \tilde{\Phi}_{1}^{n}\left(B_{m}\right)\right) \leq \mathrm{dcx}\left(\tilde{\Phi}_{2}^{n}\left(B_{1}\right), \ldots, \tilde{\Phi}_{2}^{n}\left(B_{m}\right)\right) .
$$

Since the approximation satisfies the assumption of Lemma A.2, the proof is complete.

3. We need to prove that $\mathrm{E}\left(f\left(\bar{\Phi}_{1}\left(A_{1}\right), \ldots, \bar{\Phi}_{1}\left(A_{n}\right)\right)\right) \leq \mathrm{E}\left(f\left(\bar{\Phi}_{2}\left(A_{1}\right), \ldots, \bar{\Phi}_{1}\left(A_{n}\right)\right)\right)$ for dcx, idc, and idcv functions $f$ and mutually disjoint $A_{k}, k=1, \ldots, n$; cf. Proposition 3.1. Note that, given $\Phi\left(A_{k}\right)=n_{k}$, we have $\bar{\Phi}\left(A_{k}\right)=\sum_{i=1}^{n_{k}} Z_{i}^{k}$, where the $Z_{i}^{k}$ are i.i.d. copies of the Bernoulli thinning variable. Thus, the result follows from the first statement of Lemma A.3.

4. Using the following facts from [28]:

$$
\begin{aligned}
& X \leq_{\mathrm{dcx}} Y \quad \Longrightarrow \quad X+Z \leq_{\mathrm{dcx}} Y+Z, \\
& X \leq_{\mathrm{idcx}} Y \quad \Longrightarrow X+Z \leq_{\mathrm{idcx}} Y+Z, \\
& X \leq_{\mathrm{idcv}} Y \quad \Longrightarrow \quad X+Z \leq_{\mathrm{idcv}} Y+Z,
\end{aligned}
$$

provided that $Z$ is independent of $X$ and $Y$, we can easily show that $\Lambda_{1}+\Lambda_{1}^{\prime} \leq_{\mathrm{dcx}} \Lambda_{2}+\Lambda_{1}^{\prime}$, $\Lambda_{1}+\Lambda_{1}^{\prime} \leq_{\text {idcx }} \Lambda_{2}+\Lambda_{1}^{\prime}$, and $\Lambda_{1}+\Lambda_{1}^{\prime} \leq$ idev $\Lambda_{2}+\Lambda_{1}^{\prime}$, assuming that $\Lambda_{1}^{\prime}$ is independent of $\Lambda_{2}$. The same argument shows that $\Lambda_{2}+\Lambda_{1}^{\prime} \leq_{\text {dcx }} \Lambda_{2}+\Lambda_{2}^{\prime}, \Lambda_{2}+\Lambda_{1}^{\prime} \leq_{\text {idcx }} \Lambda_{2}+\Lambda_{2}^{\prime}$, and $\Lambda_{2}+\Lambda_{1}^{\prime} \leq_{\text {idev }} \Lambda_{2}+\Lambda_{2}^{\prime}$. The result follows by the transitivity of the order.

5. This result follows easily from Lemma A.2, using an increasing approximation of $\mathbb{E}$ by bounded Borel subsets. 


\subsection{Impact on higher-order properties}

We now state some results involving the ordering of moments of random measures and draw some conclusions concerning the so-called second-order properties. These properties make it possible to characterize the clustering in PPs.

By the $k$ th power of the random measure $\Lambda$, we mean a random measure $\Lambda^{k}$ on the product space $\mathbb{E}^{k}$ given by $\Lambda^{k}\left(A_{1} \times \cdots \times A_{k}\right)=\prod_{j=1}^{k} \Lambda\left(A_{j}\right)$. Its expectation, $\alpha^{k}(\cdot)=\mathrm{E}\left(\Lambda^{k}(\cdot)\right)$, is called the $k$ th moment measure. The first moment measure, $\alpha(\cdot)=\alpha^{1}(\cdot)$, is called the mean measure.

Proposition 3.3. Consider two random measures, $\Lambda_{1}$ and $\Lambda_{2}$, such that $\Lambda_{1} \leq \mathrm{idcx} \Lambda_{2}$. Then $\Lambda_{1}^{k} \leq_{\mathrm{idcx}} \Lambda_{2}^{k}$ and $\alpha_{1}^{k}(\cdot) \leq \alpha_{2}^{k}(\cdot)$. Moreover, if $\Lambda_{1} \leq_{\mathrm{dcx}} \Lambda_{2}$ then $\alpha_{1}(\cdot)=\alpha_{2}(\cdot)$.

Proof. By the standard arguments, we can approximate any bounded Borel subsets $C_{i}, i=$ $1, \ldots, n$, in $\mathbb{E}^{k}$ by increasing unions of rectangles. By Lemma A.2 and using a similar argument as in the proof of Proposition 3.1 about the composition of idcx and idl functions, to prove the first statement, it is enough to show the respective inequality for the idcx function $f: \mathbb{R}^{n} \rightarrow \mathbb{R}$ taken on the values of the moment measures on $n$ rectangles in $\mathbb{E}^{k}$. In this context, consider the function $g: \mathbb{R}^{m} \rightarrow \mathbb{R}$, given by

$$
g\left(y_{1}, \ldots, y_{m}\right)=f\left(\prod_{j \in J_{1}} y_{j}, \ldots, \prod_{j \in J_{n}} y_{j}\right),
$$

where $J_{1}, \ldots, J_{n}$ are $k$-element subsets of the set $\{1, \ldots, m\}$. Note that, for nonnegative arguments, if $f$ is idcx then $g$ is idcx.

The second statement follows easily from the first statement by the fact that $f(x)=x$ is idcx. For the first moment (mean measure), note that both $f(x)=x$ and $f(x)=-x$ are dcx.

We now explore the relation between dcx ordering and clustering of points in a PP. One of the most popular functions for the analysis of this effect is Ripley's $K$ function, $K(r)$ (reduced second moment function); see [35, p. 129]. Assume that $\Phi$ is a stationary PP on $\mathbb{R}^{d}$ with finite intensity $\lambda=\alpha(B)$, where $B$ is a bounded Borel subset of Lebesgue measure 1 . Then

$$
K(r)=\frac{1}{\lambda|G|} \mathrm{E}\left(\sum_{X_{i} \in \Phi \cap G}\left(\Phi\left(B_{X_{i}}(r)\right)-1\right)\right),
$$

where $B_{x}(r)$ is the ball centered at $x$ with radius $r$ and $|G|$ denotes the Lebesgue measure of a bounded Borel subset $G$; owing to stationarity, the definition does not depend on the choice of $G$.

Proposition 3.4. Consider two stationary PPs $\Phi_{i}, i=1$, 2, with the same finite intensity, and denote by $K_{i}(r)$ their Ripley $K$ functions. If $\Phi_{1} \leq \mathrm{dcx} \Phi_{2}$ then $K_{1}(\cdot) \leq K_{2}(\cdot)$.

Proof. Let $I_{i}=\mathrm{E}\left(\sum_{X_{j} \in \Phi_{i} \cap G}\left(\Phi_{i}\left(B_{X_{j}}(r)\right)-1\right)\right), i=1,2$. By the equality of mean measures (Proposition 3.3), it is enough to prove that $I_{1} \leq I_{2}$. Note that $I_{i}$ can be written as the value of some shot noise evaluated with respect to $\Phi_{i}^{2}$, the second product of the PP:

$$
I_{i}=\sum_{X_{j}, X_{k} \in \Phi_{i}} 1\left[X_{j} \in G\right] \mathbf{1}\left[0<\left|X_{k}-X_{j}\right| \leq r\right] .
$$

Thus, the result follows from Proposition 3.3 and Theorem 2.1. 
Another useful characteristic is the pair correlation function defined on $\mathbb{R}^{2}$ as $g(x, y)=$ $\rho_{2}(x, y) / \rho_{1}(x) \rho_{1}(y)$, where $\rho_{k}$ is the $k$ th product intensity, equal (outside the diagonals) to the density of the $k$ th moment measure $\alpha^{k}$ with respect to the Lebesgue measure.

We avoid the discussion of questions relating to existence, etc. Corollary 3.1, below, follows from Proposition 3.3.

Corollary 3.1. Consider two PPs, $\Phi_{1}$ and $\Phi_{2}$, such that $\Phi_{1} \leq_{\mathrm{dcx}} \Phi_{2}$. Then their respective pair correlation functions satisfy $g_{1}(x, y) \leq g_{2}(x, y)$ almost everywhere with respect to the Lebesgue measure.

\subsection{Impact on Palm measures}

For the following definitions and results regarding Palm distributions of random measures, see [16, Section 10].

Definition 3.1. For a fixed measurable $f$ such that $0<\mathrm{E}\left(\int_{\mathbb{E}} f(x) \Lambda(\mathrm{d} x)\right)<\infty$, the $f$-mixed Palm version of $\Lambda$, denoted by $\Lambda_{f} \in \mathbb{M}$, is defined as having the distribution

$$
\mathrm{P}\left(\Lambda_{f} \in M\right)=\frac{\mathrm{E}\left(\int_{\mathbb{E}} f(x) \Lambda(\mathrm{d} x) \mathbf{1}[\Lambda \in M]\right)}{\mathrm{E}\left(\int_{\mathbb{E}} f(x) \Lambda(\mathrm{d} x)\right)}, \quad M \in \mathcal{M} .
$$

In the case where $\Lambda$ (say on the Euclidean space $\mathbb{E}=\mathbb{R}^{d}$ ) has a density $\{\lambda(x)\}_{x \in \mathbb{R}^{d}}$, we define, for each $x \in \mathbb{R}^{d}$, the Palm version $\Lambda_{x}$ of $\Lambda$ by

$$
\mathrm{P}\left(\Lambda_{x} \in M\right)=\frac{\mathrm{E}(\lambda(x) \mathbf{1}[\Lambda \in M])}{\mathrm{E}(\lambda(x))}, \quad M \in \mathcal{M} .
$$

Palm versions $\Lambda_{x}$ can be defined for a general random measure via some Radon-Nikodym derivatives. However, we shall state our result for $\Lambda_{x}$ as defined above as well as for mixed Palm versions $\Lambda_{f}$ in order to avoid the arbitrariness related to the nonuniqueness of Radon-Nikodym derivatives.

Proposition 3.5. Suppose that $\Lambda_{i}, i=1,2$, are random measures.

1. If $\Lambda_{1} \leq_{\mathrm{dcx}} \Lambda_{2}$ then $\left(\Lambda_{1}\right)_{f} \leq_{\mathrm{idcx}}\left(\Lambda_{2}\right)_{f}$ for any nonnegative measurable function $f$ such that $0<\int_{\mathbb{E}} f(x) \alpha(\mathrm{d} x)<\infty$, where $\alpha$ is the (common) mean measure of $\Lambda_{i}, i=1,2$.

2. Suppose that $\Lambda_{i}$ has locally finite mean measure and, a.s., locally Riemann integrable density $\lambda_{i}, i=1$, 2. If $\left\{\lambda_{1}(x)\right\} \leq_{\mathrm{dcx}}\left\{\lambda_{2}(x)\right\}$ then $\Lambda_{1} \leq_{\mathrm{dcx}} \Lambda_{2}$ and, for every $x \in \mathbb{R}^{d}$, $\left(\Lambda_{1}\right)_{x} \leq_{\mathrm{idcx}}\left(\Lambda_{2}\right)_{x}$

Proof. 1. Let $I_{i}=\int_{\mathbb{E}} f(x) \Lambda_{i}(\mathrm{~d} x), i=1,2$. By Proposition 3.3, $\Lambda_{1} \leq_{\mathrm{dcx}} \Lambda_{2}$ implies that the mean measures are equal and, thus, $\mathrm{E}\left(I_{1}\right)=\mathrm{E}\left(I_{2}\right)$. It remains to prove that

$$
\mathrm{E}\left(g\left(\Lambda_{1}\left(B_{1}\right), \ldots, \Lambda_{1}\left(B_{n}\right)\right) I_{1}\right) \leq \mathrm{E}\left(g\left(\Lambda_{2}\left(B_{1}\right), \ldots, \Lambda_{2}\left(B_{n}\right)\right) I_{2}\right)
$$

for an idcx function $g$. This follows from Theorem 2.1 and the fact that

$$
h\left(x_{0}, x\right)=x_{0} g(x): \mathbb{R}^{n+1} \rightarrow \mathbb{R}
$$

is idcx, for nonnegative argument $x_{0}$. 
2. The first part follows immediately from the second statement of Lemma A.4. For the second part, use the same fact about $h\left(x_{0}, x\right)=x_{0} g(x)$ as above.

Remark 3.1. Compared to earlier results where dcx ordering led to dcx ordering, one might tend to believe that the loss here (as dcx implies idcx only) is more technical. However, the following illustrates that it is natural to expect so: consider a Poisson PP $\Phi$ and its (deterministic) intensity measure $\alpha(\cdot)$ (i.e. its mean measure $\alpha(\cdot)=\mathrm{E}(\Phi(\cdot))$ ). Using the complete independence property of the Poisson PP and the fact that each dcx function is componentwise convex, we can show that, for disjoint, bounded Borel subsets $A_{1}, \ldots, A_{n}$ and any dex function $f, f\left(\alpha\left(A_{1}\right), \ldots, \alpha\left(A_{n}\right)\right) \leq \mathrm{E}\left(f\left(\Phi\left(A_{1}\right), \ldots, \Phi\left(A_{n}\right)\right)\right)$. Thus, $\alpha \leq_{\mathrm{dcx}} \Phi$. It is easy to see that $\alpha_{f}(\cdot)=\alpha(\cdot)$ (the mixed Palm version of a deterministic measure is equal to the original measure). Take $f(x)=\mathbf{1}[x \in A]$ for some bounded Borel subset $A$. Then $\mathrm{E}\left(\Phi_{f}(A)\right)=\mathrm{E}\left((\Phi(A))^{2}\right) / \alpha(A)=\alpha(A)+1$ since $\Phi(A)$ is a Poisson RV. Thus, $\alpha_{f}(A)<\mathrm{E}\left(\Phi_{f}(A)\right)$, disproving $\alpha_{f}(A) \leq_{\mathrm{dcx}} \Phi_{f}(A)$. Another counterexample involving Poisson-Poisson cluster PPs will be given in Remark 5.2, below.

\subsection{Cox point processes}

We now consider Cox PPs (see, e.g. [35, Chapter 5.2]), also known as doubly stochastic Poisson PPs, which constitute a rich class often used to model patterns that exhibit more clustering than in Poisson PPs.

Recall that a $\operatorname{Cox}(\Lambda) \operatorname{PP} \Phi_{\Lambda}$ on $\mathbb{E}$ generated by the random intensity measure $\Lambda(\cdot)$ on $\mathbb{E}$ is defined as having the property that $\Phi_{\Lambda}$ conditioned on $\Lambda(\cdot)$ is a Poisson PP with intensity $\Lambda(\cdot)$. Note that Cox PPs may arise as a result of a transformation of a random measure.

We can easily show that this operation preserves our orders.

Proposition 3.6. Consider two ordered random measures, $\Lambda_{1}$ and $\Lambda_{2}$, such that $\Lambda_{1} \leq_{\mathrm{dcx}} \Lambda_{2}$,

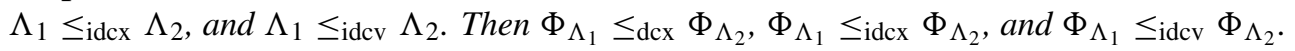

Proof. Taking a dcx, idcx, or idcv function $\phi$, assuming (by Proposition 3.1) mutually disjoint, bounded Borel subsets $A_{k}, k=1, \ldots, n$, and using the definition of Cox PPs and the second statement of Lemma A.3, we show that, for $i=1,2$, the conditional expectation

$$
\mathrm{E}\left(\phi\left(\Phi_{\Lambda_{i}}\left(A_{1}\right), \ldots, \Phi_{\Lambda_{i}}\left(A_{n}\right)\right) \mid \Lambda_{i}\right)
$$

given the intensity measure $\Lambda_{i}$, is a dcx, idcx, or, respectively, idcv function of $\left(\Lambda_{i}\left(A_{1}\right), \ldots\right.$, $\Lambda_{i}\left(A_{n}\right)$ ). The result then follows from the assumption of the measures $\Lambda_{i}$ being dcx-ordered.

We now show, using Theorem 2.1, that dcx, idcx, and idcv ordering of Cox intensity measures is preserved by independent (not necessarily identically distributed) marking and thinning, as well as independent displacement of points of the PP.

By independent marking of the PP $\Phi$ on $\mathbb{E}$ with marks on some LCSC space $\mathbb{E}^{\prime}$ we mean a PP $\tilde{\Phi}=\sum_{i} \varepsilon_{\left(x_{i}, Z_{i}\right)}$ such that, given $\Phi=\sum_{i} \varepsilon_{x_{i}}, Z_{i}$ are independent random elements in $\mathbb{E}^{\prime}$, with distribution $\mathrm{P}\left(Z_{i} \in \cdot \mid \Phi=\sum_{i} \varepsilon_{x_{i}}\right)=F_{x_{i}}(\cdot)$ given by some probability (mark) kernel $F_{x}(\cdot)$ from $\mathbb{E}$ to $\mathbb{E}^{\prime}$. The fact that $F_{x}(\cdot)$ may depend on $x$ (in contrast to i.i.d. marking) is sometimes emphasized by calling $\tilde{\Phi}$ a 'position-dependent' marking. Independent thinning can be seen as the projection on $\mathbb{E}$ of the subset $\tilde{\Phi}(\cdot,\{1\})$ of the independently marked PP $\tilde{\Phi}$, where the marks $Z_{i} \in\{0,1\}=\mathbb{E}^{\prime}$ are independent Bernoulli thinning variables $Z_{i}=Z_{i}(x)$, whose distributions may be dependent on $x_{i}$. Similarly, the projection of an independently marked PP $\tilde{\Phi}=\sum_{i} \varepsilon_{\left(x_{i}, Z_{i}\right)}$ on the space of marks $\mathbb{E}^{\prime}$, i.e. the $\tilde{\Phi}(\mathbb{E} \times \cdot)=\sum_{i} \varepsilon_{Z_{i}}$, can be seen as the 
independent displacement of points of $\Phi$ to the space $\mathbb{E}^{\prime}$. Special examples are i.i.d. shifts of points in the Euclidean space, when $Z_{i}=x_{i}+Y_{i}$, where the $Y_{i}$ are i.i.d.

Proposition 3.7. Suppose that $\Phi_{i}, i=1,2$, are two $\operatorname{Cox}\left(\Lambda_{i}\right) P P$ s. Assume that their intensity measures are ordered $\Lambda_{1} \leq_{\mathrm{dcx}} \Lambda_{2}, \Lambda_{1} \leq_{\mathrm{idcx}} \Lambda_{2}$, and $\Lambda_{1} \leq_{\mathrm{idcv}} \Lambda_{2}$. Let $\tilde{\Phi}_{i}, i=1,2$, be the corresponding independently marked PPs with the same mark kernel $F_{x}(\cdot)$. Then $\tilde{\Phi}_{1} \leq_{\mathrm{dcx}} \tilde{\Phi}_{2}$, $\tilde{\Phi}_{1} \leq_{\text {idcx }} \tilde{\Phi}_{2}$, and $\tilde{\Phi}_{1} \leq_{\text {idcv }} \tilde{\Phi}_{2}$.

From Proposition 3.7, and using the last statement of Proposition 3.2, Corollary 3.2, below, follows immediately.

Corollary 3.2. Independent thinning and displacement of points preserves the $d c x$, idcx, and idcv orders of the intensities of Cox PPs.

Proof of Proposition 3.7. Let $\Phi_{i}$ be $\operatorname{Cox}\left(\Lambda_{i}\right), i=1,2$. Assume that $\Lambda_{1} \leq_{\mathrm{dcx}} \Lambda_{2}, \Lambda_{1} \leq_{\mathrm{idcx}}$ $\Lambda_{2}$, and $\Lambda_{1} \leq_{\text {idcv }} \Lambda_{2}$. It is known that independent marking of a $\operatorname{Cox}\left(\Lambda_{i}\right) \operatorname{PP}$ is a $\operatorname{Cox}\left(\tilde{\Lambda}_{i}\right)$ PP with intensity measure $\tilde{\Lambda}_{i}$ on $\mathbb{E} \times \mathbb{E}^{\prime}$ given by $\tilde{\Lambda}_{i}(\cdot)=\int_{\mathbb{E}} \int_{\mathbb{E}^{\prime}} \mathbf{1}[(x, y) \in \cdot] F_{x}(\mathrm{~d} y) \Lambda_{i}(\mathrm{~d} x)$; cf. [35, Sections 4.2 and 5.2]. Let $S$ be the family of bounded Borel subsets in $\mathbb{E} \times \mathbb{E}^{\prime} ;$ for $x \in \mathbb{E}$ and bounded Borel subsets $C \subset \mathbb{E} \times \mathbb{E}^{\prime}$, consider $h(x, C)=\int_{\mathbb{E}^{\prime}} \mathbf{1}[(x, y) \in C] F_{x}(\mathrm{~d} y)$. Then the integral shot noise $V_{\Lambda_{i}}(C)=\int_{\mathbb{E}} h(x, C) \Lambda_{i}(\mathrm{~d} x)$ satisfies $V_{\Lambda_{i}}(C)=\tilde{\Lambda}_{i}(C)$ for all bounded Borel subsets $C$. Thus, by Theorem 2.1, $\tilde{\Lambda}_{1} \leq_{\mathrm{dxc}} \tilde{\Lambda}_{2}, \tilde{\Lambda}_{1} \leq_{\mathrm{idxc}} \tilde{\Lambda}_{2}$, and $\tilde{\Lambda}_{1} \leq_{\mathrm{idxv}} \tilde{\Lambda}_{2}$, and the result follows from Proposition 3.6.

If $\Lambda(\cdot) \in \mathbb{M}\left(\mathbb{R}^{d}\right)$ a.s. has a density $\{\lambda(x)\}_{x \in \mathbb{R}^{d}}$ with respect to the Lebesgue measure then the density is referred to as the intensity field of the Cox PP, which in this case will be called the $\operatorname{Cox}(\lambda)$ PP and denoted by $\Phi_{\lambda}$.

It is known that the Cox PP is over-dispersed with respect to the Poisson PP, i.e. $\operatorname{var}\left(\Phi_{1}(B)\right) \leq$ $\operatorname{var}\left(\Phi_{2}(B)\right)$, where $\Phi_{1}$ and $\Phi_{2}$ are respectively Poisson and Cox PPs with the same mean measure. Hence, it is clear that a Cox PP can only be greater than a Poisson PP with the same mean measure in the dcx order. Indeed, in Section 5 we show several examples where this stronger result holds, namely Cox PPs that are dcx-ordered (larger) with respect to the corresponding Poisson PPs, as well as Cox PPs dcx-ordered with respect to each other.

\subsection{Alternative definition of the dcx order}

We viewed a random measure as a random field and defined ordering from this viewpoint. Alternatively, we can consider a random measure as an element of the space of Radon measures $\mathbb{M}$ and define ordering between two $\mathbb{M}$-valued random elements. This can be done once we define what is a dcx function on $\mathbb{M}$. The dcx order can be defined on more general spaces; Meester and Shanthikumar [21] extended the notion of dcx ordering to lattice-ordered Abelian semigroups with some compatibility conditions between the lattice structure and the Abelian structure $\left(\mathrm{LOAS}^{+}\right)$. The space $\mathbb{M}$ can be equipped with the following lattice and algebraic structure. Consider the following partial order and addition operation on $\mathbb{M}$ : for $\mu, v \in \mathbb{M}$, we say that $\mu \leq v$ if $\mu(B) \leq v(B)$ for all bounded Borel subsets $B$ in $\mathbb{E}$ and $(\mu+v)(B)=$ $\mu(B)+v(B)$. Under this definition, the space $\mathbb{M}$ forms an $\mathrm{LOAS}^{+}$, as required by [21]. Then we can define a dex function on $\mathbb{M}$ as in Definition 2.1. Call it a dcx ${ }^{1}$ function. This gives rise to the $\mathrm{dcx}^{1}$ order of random measures, analogously to the first part of Definition 2.2.

Now we have two reasonable definitions for the ordering of random measures. It is easy to see that $\mathrm{dcx}^{1}$ ordering implies dcx ordering. In light of Example 5.1.7 of [28], existence of a counterexample to the converse looks plausible, though we failed in our attempts to construct one. However, the result of [3] proves that convex ordering of real-valued stochastic processes 
$\left\{X_{n}\right\}_{n \in \mathbb{N}}$ implies continuous, convex ordering of the corresponding elements of the infinitedimensional Euclidean spaces $\mathbb{R}^{\mathbb{N}}$. This suggests that the dcx order of random measures may imply a dcx ${ }^{1 *}$ order induced by some subclass of $\mathrm{dcx}^{1}$ functionals of random measures, which are regular in some sense. Leaving this general question as an open problem, we only remark that the integral shot noise fields studied in the next section can be seen as some particular class of functionals of random measures, which are $\mathrm{dcx}^{1}$ (in fact, linear on $\mathbb{M}$ ) and regular enough for their means to satisfy the required inequality, provided that the random measures are dcx-ordered. It is thus natural to have them in the suggested dcx ${ }^{1 *}$ class.

Recall also that, for the strong order of PPs, there is full equivalence between these two definitions, and both imply the possibility of a coupling of the ordered PPs such that the smaller one is a.s. a subset of the larger one; cf. [31].

\section{Ordering of shot noise fields}

In this section we prove Theorem 2.1, which concerns the dcx ordering of integral shot noise fields and is the main result of this paper. We also consider the so-called extremal shot noise fields.

\subsection{Integral shot noise fields}

Usually shot noise fields are defined for PPs by the following sum (thus, they are sometimes called additive shot noise fields): $V_{\Phi}(y)=\sum_{X_{n} \in \Phi} h\left(X_{n}, y\right)$, where $\Phi=\sum_{n} \varepsilon_{X_{n}}$ and $h$ is a nonnegative response function. In Definition 2.4 we made a significant but natural generalization of this definition. It is fairly clear as to why we call this generalization an integral shot noise field. The extension to unbounded response functions is not just a mathematical generalization. It will provide us with a simple proof of the ordering of extremal shot noise fields for PPs.

The following proof of Theorem 2.1 is inspired by [23].

Proof of Theorem 2.1. We prove the second statement first. The necessary modifications for the proof of the first statement will be indicated later on.

We need to show that $\left(V^{1}\left(y_{1}\right), \ldots, V^{1}\left(y_{m}\right)\right) \leq_{\mathrm{dcx}}\left(V^{2}\left(y_{1}\right), \ldots, V^{2}\left(y_{m}\right)\right)$ for $y_{i} \in S$, $1 \leq i \leq m$, and $V^{j}(\cdot)=V_{\Lambda_{j}}(\cdot), j=1,2$. The proof relies on the construction of two sequences of random vectors $\left(V_{k}^{j}\left(y_{1}\right), \ldots, V_{k}^{j}\left(y_{m}\right)\right), k=1,2, \ldots, j=1,2$, satisfying the assumptions of Lemma A.2.

Choose an increasing sequence of compact sets $K_{k}, k \geq 1$, in $\mathbb{E}$ such that $K_{k} \nearrow \mathbb{E}$. Since $h$ is measurable in its first argument, we know that there exists a sequence of simple functions $h_{k}\left(\cdot, y_{i}\right), k \in \mathbb{N}$, such that, as $k \rightarrow \infty, h_{k}\left(\cdot, y_{i}\right) \uparrow h\left(\cdot, y_{i}\right)$ for $1 \leq i \leq m$. They can be written down explicitly as follows:

$$
\begin{aligned}
h_{k}\left(\cdot, y_{i}\right)= & \gamma_{k} \mathbf{1}\left[\left\{x \in K_{k}: h\left(x, y_{i}\right)=\infty\right\}\right] \\
& +\sum_{n=1}^{\gamma_{k}} \frac{n-1}{2^{k}} \mathbf{1}\left[\left\{x \in K_{k}: \frac{n-1}{2^{k}} \leq h\left(x, y_{i}\right)<\frac{n}{2^{k}}\right\}\right](\cdot)
\end{aligned}
$$

for $1 \leq i \leq m$, where $\gamma_{k}=k 2^{k}$. Set $I_{k n}^{i}=\left\{x \in K_{k}:(n-1) / 2^{k} \leq h\left(x, y_{i}\right)<n / 2^{k}\right\}$ and $I_{k \infty}^{i}=\left\{x \in K_{k}: h\left(x, y_{i}\right)=\infty\right\}$ for $1 \leq i \leq m$ and $1 \leq n \leq \gamma_{k}$. Note that all the $I_{k n}^{i}, n=1, \ldots, \infty$, are bounded Borel subsets and the sequence of random vectors we are looking for is

$$
V_{k}^{j}\left(y_{i}\right)=\int_{\mathbb{E}} h_{k}\left(x, y_{i}\right) \Lambda_{j}(\mathrm{~d} x)=\gamma_{k} \Lambda_{j}\left(I_{k \infty}^{i}\right)+\sum_{n=1}^{\gamma_{k}} \frac{n-1}{2^{k}} \Lambda_{j}\left(I_{k n}^{i}\right)
$$


for $j=1,2$. By the definition of the integral, it is clear that, for $j=1,2$, as $k \rightarrow \infty$, $\left(V_{k}^{j}\left(y_{1}\right), \ldots, V_{k}^{j}\left(y_{m}\right)\right) \uparrow\left(V^{j}\left(y_{1}\right), \ldots, V^{j}\left(y_{m}\right)\right)$ a.s. and, hence, in distribution. By the monotone convergence theorem, the expectations, which are finite by the assumption, also converge. What remains to prove is that, for each $k \in \mathbb{N}$, the vectors are dcx-ordered.

Fix $k \in \mathbb{N}$. Now observe that, for $j=1,2$ and $i=1, \ldots, m$, the $V_{k}^{j}\left(y_{i}\right)$ are increasing linear functions of the vectors $\left(\Lambda_{j}\left(I_{k n}^{i}\right): n=1, \ldots, \gamma_{k}, \infty\right), j=1,2$. The latter are dexordered by the assumptions. Also, since composition of dex with increasing linear functions is dex, it follows that $\left(V_{k}^{1}\left(y_{1}\right), \ldots, V_{k}^{1}\left(y_{m}\right)\right) \leq_{\mathrm{dcx}}\left(V_{k}^{2}\left(y_{1}\right), \ldots, V_{k}^{2}\left(y_{m}\right)\right)$.

We now prove the first statement. For vectors $\left(V_{k}^{j}\left(y_{1}\right), \ldots, V_{k}^{j}\left(y_{m}\right)\right), k=1,2, \ldots$, $j=1,2$, defined as above, $f\left(V_{k}^{j}\left(y_{1}\right), \ldots, V_{k}^{j}\left(y_{m}\right)\right) \uparrow f\left(V^{j}\left(y_{1}\right), \ldots, V^{j}\left(y_{m}\right)\right)$ a.s. for idcx and idev functions $f$, and, hence, $\mathrm{E}\left(f\left(V_{k}^{j}\left(y_{1}\right), \ldots, V_{k}^{j}\left(y_{m}\right)\right)\right) \uparrow \mathrm{E}\left(f\left(V^{j}\left(y_{1}\right), \ldots, V^{j}\left(y_{m}\right)\right)\right)$, $j=1,2$. The proof is complete by noting that

$$
\mathrm{E}\left(f\left(V_{k}^{1}\left(y_{1}\right), \ldots, V_{k}^{1}\left(y_{m}\right)\right)\right) \leq \mathrm{E}\left(f\left(V_{k}^{2}\left(y_{1}\right), \ldots, V_{k}^{2}\left(y_{m}\right)\right)\right)
$$

for all $k \geq 1$ and idex and idev functions $f$.

\subsection{Extremal shot noise fields}

We now recall the definition of the extremal shot noise, first introduced in [11].

Definition 4.1. Let $S$ be any set, and let $\mathbb{E}$ be an LCSC space. Given a PP $\Phi$ on $\mathbb{E}$ and a measurable (in the first variable alone) response function $h(x, y): \mathbb{E} \times S \rightarrow \mathbb{R}$, the extremal shot noise field is defined as

$$
U_{\Phi}(y)=\sup _{X_{i} \in \Phi}\left\{h\left(X_{i}, y\right)\right\}
$$

In order to state our result for extremal shot noise fields, we shall use the lower orthant (lo) order.

Definition 4.2. Let $X$ and $Y$ be random $\mathbb{R}^{d}$ vectors. We say that $X \leq_{\text {lo }} Y$ if $\mathrm{P}(X \leq t) \geq$ $\mathrm{P}(Y \leq t)$ for every $t \in \mathbb{R}^{d}$.

On the real line, this is the same as the st order (i.e. when $\mathfrak{F}$ consists of increasing functions), but in higher dimensions it is different. Obviously, the st order implies the lo order, and examples of random vectors which are lo-ordered but not st-ordered are known; see [28, Chapter 3]. Thus, it is clear that the following proposition is a generalization of the corresponding one-dimensional result in [23], where the proof method was similar to the proof of the ordering of integral shot noise fields. We shall give a much simpler proof using the already proved result.

Proposition 4.1. Let $\Phi_{1} \leq_{\text {idcv }} \Phi_{2}$. Then $\left\{U_{\Phi_{1}}(y)\right\}_{y \in S} \leq_{\text {lo }}\left\{U_{\Phi_{2}}(y)\right\}_{y \in S}$.

Proof. The probability distribution function of the extremal shot noise can be expressed in terms of the Laplace transform of some additive shot noise field. Let $\left\{x_{1}, \ldots, x_{m}\right\} \subset S$ and $\left(a_{1}, \ldots, a_{m}\right) \in R^{m}$. Then

$$
\begin{aligned}
\mathrm{P}\left(U\left(y_{i}\right) \leq a_{i}, 1 \leq i \leq m\right) & =\mathrm{E}\left(\prod_{i} \mathbf{1}\left[\sup _{n}\left\{h\left(X_{n}, y_{i}\right) \leq a_{i}\right\}\right]\right) \\
& =\mathrm{E}\left(\prod_{i} \prod_{n} \mathbf{1}\left[h\left(X_{n}, y_{i}\right) \leq a_{i}\right]\right) \\
& =\mathrm{E}\left(\prod_{i} \prod_{n} \exp \left(\log \mathbf{1}\left[h\left(X_{n}, y_{i}\right) \leq a_{i}\right]\right)\right)
\end{aligned}
$$




$$
\begin{aligned}
& =\mathrm{E}\left(\prod_{i} \exp \left(-\sum_{n}-\log \mathbf{1}\left[h\left(X_{n}, y_{i}\right) \leq a_{i}\right]\right)\right) \\
& =\mathrm{E}\left(\exp \left(-\sum_{i} \hat{U}\left(y_{i}\right)\right)\right),
\end{aligned}
$$

where $\hat{U}\left(y_{i}\right)=\sum_{n}-\log \mathbf{1}\left[h\left(X_{n}, y_{i}\right) \leq a_{i}\right]$ is an additive shot noise with response function taking values in $[0, \infty]$. The response function is clearly nonnegative and measurable. The function $f\left(x_{1}, \ldots, x_{m}\right)=\exp \left(-\sum_{i} x_{i}\right)$ is a ddcx function on $(-\infty, \infty]$. The result follows by the first statement of Theorem 2.1.

The extremal shot noise field can be used to define the Boolean model. Given a (generic) random closed set (see [35, Chapter 6]) $G$, let $h((x, G), y)=\mathbf{1}[y \in x+G]$.

Definition 4.3. For a PP of germs $\Phi$ and a typical grain $G$, the random set $C(\Phi, G)=$ $\left\{y: U_{\tilde{\Phi}}(y)>0\right\}$ is called a Boolean model, where $\tilde{\Phi}=\sum_{i} \varepsilon_{\left(X_{i}, G_{i}\right)}$ is the i.i.d. marking of $\Phi$ with mark distribution equal to that of $G$.

We call $G$ a fixed grain if there exists a closed set $B$ such that $G=B$ a.s. We shall demonstrate in Section 6.1 how to obtain comparison results for the Boolean model using the results of this section.

\section{Examples of dcx-ordered measures and point processes}

In this section we provide some examples of dcx-ordered measures and PPs on the Euclidean space $\mathbb{E}=\mathbb{R}^{d}$. The examples are intended to be illustrative and not encyclopaedic. The purpose of the examples is to show that there are dcx-ordered PPs, as well as to demonstrate some methods to prove that two PPs are dcx-ordered. Many of the examples seem to indicate that PPs higher in dcx order cluster more, at least for Cox PPs.

\subsection{Ising-Poisson cluster point processes}

Let $\{\lambda(s)\}_{s \in \mathbb{R}^{d}}$ be a stationary random intensity field. Define two new fields, one which is random but constant in space $\left\{\lambda_{m}(s)=\lambda(0)\right\}$ and another one which is deterministic constant $\left\{\lambda_{h}(s)=\mathrm{E}(\lambda(0))\right\}$. $\operatorname{Cox}\left(\lambda_{m}\right)$ is known as a mixed Poisson PP, and $\operatorname{Cox}\left(\lambda_{h}\right)$ is just the wellknown homogeneous Poisson PP. Denote the random intensity measures of the Cox, mixed, and homogeneous Poisson PPs, by $\Lambda, \Lambda_{m}$, and $\Lambda_{h}$, respectively (i.e. $\Lambda(\mathrm{d} x)=\lambda(x) \mathrm{d} x$, etc.). It was proved in [23] that $\Lambda \leq_{\mathrm{dcx}} \Lambda_{m}$ and, when $\{\lambda(s)\}$ is a conditionally increasing field, that $\Lambda_{h} \leq_{\mathrm{dcx}} \Lambda$. Recall that a random field $\{X(s)\}$ is a conditionally increasing field if, for any $k$ and $s_{1}, \ldots, s_{k} \in \mathbb{R}^{d}$, the expectation $\mathrm{E}\left(f\left(X\left(s_{1}\right)\right) \mid X\left(s_{j}\right)=a_{j}\right.$ for all $\left.2 \leq j \leq k\right)$ is increasing in $a_{j}$ for all increasing $f$. However, no example of a conditionally increasing field was given in [23]. Now we construct one.

Consider the $d$-dimensional lattice $\mathbb{Z}^{d}$. Let $\{X(z)\}_{z \in \mathbb{Z}^{d}}$ be i.i.d. RVs taking values in $\{+1,-1\}$. Call $\{X(z)\}$ a (random) configuration of spins. In order to obtain a stationary field, consider a random shift of the origin of $\mathbb{Z}^{d}$ to $U$ with uniform distribution on $[0,1]^{d}$ ( $U$ independent of $\{X(z)\}$ ). Let the lattice shifted by $U$ be denoted by $\mathbb{Z}_{*}^{d}$. Pick two numbers $\mu_{2} \leq \mu_{1}$. For $s \in \mathbb{R}^{d}$, define $\lambda(s)=\mu_{1} \mathbf{1}[X(\dot{s})=1]+\mu_{2} \mathbf{1}[X(\dot{s})=-1]$, where $\dot{s}$ represents the unique 'lower-left' point in $\mathbb{Z}_{*}^{d}$ nearest to $s$. The intensity field is clearly stationary. We now show that $\{\lambda(s)\}$ is conditionally increasing. Note that

$$
f(\lambda(s))=\mathbf{1}[x(\dot{s})=1]\left(f\left(\mu_{1}\right)-f\left(\mu_{2}\right)\right)+f\left(\mu_{2}\right) .
$$


From Theorem 1.2.15 of [28], it is sufficient to show the conditional increasing property conditioned on $U$, the random origin of the lattice $\mathbb{Z}_{*}^{d}$. Hence, it is enough for the Ising model to possess the following property:

$$
\begin{aligned}
& \mathrm{P}\left(X\left(z_{1}\right)=1 \mid X\left(z_{2}\right)=-1, X\left(z_{j}\right)=a_{j}, j=3, \ldots, k\right) \\
& \quad \leq \mathrm{P}\left(X\left(z_{1}\right)=1 \mid X\left(z_{2}\right)=1, X\left(z_{j}\right)=a_{j}, j=3, \ldots, k\right),
\end{aligned}
$$

where $a_{i} \in\{+1,-1\}$ and $z_{i} \in \mathbb{Z}^{d}, i=1, \ldots, k$. This follows easily from the fact that the spins are i.i.d.

We call the Cox PP generated by the above conditionally increasing field $\{\lambda(s)\}$ the IsingPoisson cluster PP. By the arguments presented in [23], it is dcx larger than the homogeneous Poisson PP with the same intensity. Note that intuitively the Ising-Poisson cluster PP 'clusters' its points more than a homogeneous Poisson PP. In what follows, we shall see more examples of cluster (Cox) PPs which are dex larger than the corresponding homogeneous Poisson PPs.

\subsection{Lévy-based Cox point processes (LCPPs)}

This class of PPs is introduced in [12]. One can find many examples of LCPPs in the abovementioned paper. In simple terms, an LCPP is a PP whose intensity field is an integral shot noise field of a Lévy basis. A random measure $L \in \mathbb{M}\left(\mathbb{R}^{d}\right)$ is said to be a nonnegative Lévy basis if

- for any sequence $\left\{A_{n}\right\}$ of disjoint, bounded Borel subsets of $\mathbb{R}^{d}, L\left(A_{n}\right)$ are independent RVs (complete independence) and $L\left(\cup A_{n}\right)=\sum L\left(A_{n}\right)$ a.s., provided that $\cup A_{n}$ is also a bounded Borel subset of $\mathbb{R}^{d}$,

- for every bounded Borel subset $A$ of $\mathbb{R}^{d}, L(A)$ is infinitely divisible.

We shall consider only nonnegative Lévy bases, even though there exist signed Lévy bases too (see [12]). Hence, we shall omit the reference to nonnegativity in what follows.

A Cox PP $\Phi$ is said to be an LCPP if its intensity field is of the form

$$
\lambda(y)=\int_{\mathbb{R}^{d}} k(x, y) L(\mathrm{~d} x),
$$

where $L$ is a Lévy basis, and the kernel $k$ is a nonnegative function such that $k(x, y)$ is a.s. integrable with respect to $L$ and $k(\cdot, y)$ is integrable with respect to the Lebesgue measure. In [12] the response function $k$ and the Lévy basis $L$ are chosen such that $\int_{B} \lambda(y) \mathrm{d} y<\infty$ a.s. for all bounded Borel subsets $B$, for which a sufficient condition is $\int_{B} E(\lambda(y)) d y<\infty$. In our considerations, in order to be able to use Lemma A.4, we shall require that $\lambda(y)$ is a.s. locally Riemann integrable.

Remark 5.1. Note that a sufficient condition for $\lambda(y)$ to be a.s. locally Riemann integrable is that $\lambda(y)$ is a.s. continuous, for which, in turn, it is enough to assume that $k$ is continuous in its second argument and that, for all $x \in \mathbb{R}^{d}$, there exist $B_{x}\left(\epsilon_{x}\right), \epsilon_{x}>0$, such that $\int_{\mathbb{R}^{d}} \sup _{z \in B_{x}\left(\epsilon_{x}\right)} k(z, y) \alpha(\mathrm{d} x)<\infty$ for all $y$, where $\alpha(B)=\mathrm{E}(L(B))$, the mean measures of the Lévy bases (cf. [1]).

Lemma 5.1. Let $L_{1}$ and $L_{2}$ be Lévy bases with mean measure $\alpha_{i}$. Let $\Phi_{i}, i=1,2$, be LCPPs with Lévy bases $L_{i}, i=1,2$, respectively.

1. $L_{1} \leq_{\mathrm{dcx}} L_{2}, L_{1} \leq_{\mathrm{idcx}} L_{2}$, and $L_{1} \leq_{\mathrm{idcv}} L_{2}$ if and only if $L_{1}(A) \leq_{\mathrm{cx}} L_{2}(A), L_{1}(A) \leq_{\mathrm{icx}}$ $L_{2}(A)$, and $L_{1}(A) \leq_{\mathrm{icv}} L_{2}(A)$, respectively, for all bounded Borel subsets $A$ of $\mathbb{R}^{d}$, 
where cx, icx, and icv respectively stand for convex, increasing convex, and increasing concave.

2. If $L_{1} \leq_{\mathrm{dcx}} L_{2}, L_{1} \leq_{\mathrm{idcx}} L_{2}$, and $L_{1} \leq_{\mathrm{idcv}} L_{2}$, then $\Phi_{1} \leq_{\mathrm{dcx}} \Phi_{2}, \Phi_{1} \leq_{\mathrm{idcx}} \Phi_{2}$, and $\Phi_{1} \leq_{\mathrm{idcv}} \Phi_{2}$, respectively, provided that the intensity fields $\lambda_{i}(y)$ of the $\Phi_{i}$ are a.s. locally Riemann integrable with these integrals, in the dcx case, having finite means.

3. $\alpha_{i} \leq_{\mathrm{dcx}} L_{i}$.

Proof. The first part is due to Proposition 3.1 and the complete independence property of Lévy bases. As for the second part, it is a simple consequence of Theorem 2.1, Lemma A.4, and Proposition 3.6. The third part follows from complete independence and Jensen's inequality.

We now give some examples of dcx-ordered Lévy bases.

Example 5.1. Let $\left\{x_{i}\right\}$ be a locally finite deterministic configuration of points in $\mathbb{R}^{d}$. Let $\left\{X_{i}^{j}\right\}_{i \geq 1}, j=1,2$, be an i.i.d. sequence of infinitely divisible RVs such that $X_{1}^{1} \leq \mathrm{cx} X_{1}^{2}$. (For example, $X_{1}^{1}$ can be the sum of two independent exponential RVs with mean $\frac{1}{2}$ and $X_{1}^{2}$ can be an exponential RV with mean 1.) Define the Lévy bases as follows:

$$
L_{j}(A)=\sum_{x_{i} \in A} X_{i}^{j},
$$

where $A$ is a bounded Borel subset of $\mathbb{R}^{d}$ and $j=1,2$. By Lemma 5.1 and the fact that $X_{1}^{1} \leq_{\mathrm{cx}} X_{1}^{2}$, it follows that $L_{1} \leq_{\mathrm{dcx}} L_{2}$.

Example 5.2. Let $\tilde{\Phi}=\sum_{i} \varepsilon_{\left(x_{i}, Z_{i}\right)}$ be a homogeneous Poisson PP on $\mathbb{R}^{d}$ independently marked by RVs $\left\{Z_{i}\right\}$ with mean $\lambda_{0}$. Consider two random measures, $\Lambda_{1}=\sum_{\left(x_{i}, Z_{i}\right) \in \tilde{\Phi}} \lambda_{0} \varepsilon_{x_{i}}$ and $\Lambda_{2}=\sum_{\left(x_{i}, Z_{i}\right) \in \tilde{\Phi}} Z_{i} \varepsilon_{x_{i}}$. Note that the $L_{i}, i=1,2$, are Lévy bases. By Lemma 5.1 and the fact that $\lambda_{0} \leq_{\mathrm{cx}} Z_{i}$, conditioning on the number of points and using the same argument as in the proof of the second statement of Proposition 3.2, we can prove that $\Lambda_{1} \leq_{\mathrm{dcx}} \Lambda_{2}$.

\subsection{Poisson-Poisson cluster point processes}

By a Poisson-Poisson cluster PP we mean an LCPP whose Lévy basis is a Poisson PP. This class deserves a separate mention due to the generality of the ordering results that are possible. For the rest of the section, assume that $h(x)$ is a nonnegative measurable function such that $\int_{\mathbb{R}^{d}} h(x) \mathrm{d} x=\lambda_{0}<\infty$.

We now give an example of a parametric family of dcx-ordered Poisson-Poisson cluster PPs. Fix $\lambda>0$. Let $\Phi_{c}, c>0$, be a family of homogeneous Poisson PPs on $\mathbb{R}^{d}$ of intensity $c \lambda$. Let a nonnegative function $h: \mathbb{R}^{d} \times \mathbb{R}^{d} \rightarrow \mathbb{R}$ be given, and consider a family of shot noise fields $\lambda_{c}(y)=\int_{\mathbb{R}^{d}}(h(x, y) / c) \Phi_{c}(\mathrm{~d} x)$, which are assumed to be a.s. locally Riemann integrable with $\int_{B} \mathrm{E}\left(\lambda_{c}(y)\right) \mathrm{d} y<\infty$ for bounded Borel subsets $B$.

Proposition 5.1. The family of shot noise fields $\left\{\lambda_{c}(y)\right\}_{y \in \mathbb{R}^{d}}$ is decreasing in the dcx order, i.e. for $0<c_{1} \leq c_{2}$, we have $\left\{\lambda_{c_{2}}(y)\right\} \leq_{\mathrm{dcx}}\left\{\lambda_{c_{1}}(y)\right\}$. Consequently, $\operatorname{Cox}\left(\lambda_{c_{2}}\right) \leq_{\mathrm{dcx}} \operatorname{Cox}\left(\lambda_{c_{1}}\right)$.

Proof. Note that $\left\{\lambda_{c}(x)\right\}$ can be seen as a shot noise field generated by the response function $h$ and the Lévy basis $L_{c}=(1 / c) \Phi_{\lambda c}$. By Lemma 5.1 and Theorem 2.1, it is enough to prove that $L_{c_{2}}(A) \leq_{\mathrm{cx}} L_{c_{1}}(A)$ for bounded Borel subsets $A$ and $c_{2}>c_{1}>0$.

Since $X \leq_{\mathrm{cx}} Y$ implies that $a X \leq_{\mathrm{cx}} a Y$ for all scalars $a>0$, it suffices to prove that $L_{c a}(A) \leq_{\mathrm{cx}} L_{a}(A)$ for bounded Borel subsets $A, c>1$, and $a>0$. This essentially boils down to proving that $N_{c a} \leq_{\mathrm{cx}} c N_{a}, c>1, a>0$, where $N_{a}$ stands for a Poisson RV with mean $a$. 
Let $\left\{X_{i}^{n}\right\}_{1 \leq i \leq n}$ and $\left\{Y_{i}^{n}\right\}_{1 \leq i \leq n}, n \geq 1$, be i.i.d. sequences of Bernoulli RVs with probabilities of success $c a / n$ and $a / n$, respectively, with $n \geq c a$. Let $X^{n}=\sum_{i=1}^{n} X_{i}^{n}$ and $Y^{n}=\sum_{i=1}^{n} c Y_{i}^{n}$. It is well known that $X^{n}$ and $Y^{n}$ converge weakly to $N_{c a}$ and $N_{a}$, respectively, as $n \rightarrow \infty$. As the convex order preserves weak convergence, we need only prove that $X^{n} \leq_{\mathrm{cx}} c Y^{n}$. By the independence of summands, it is enough to prove that $X_{i}^{n} \leq_{\mathrm{cx}} c Y_{i}^{n}$, which we shall do in what follows. Let $f$ be a convex and differentiable function. Define

$$
g(c):=\mathrm{E}\left(f\left(X_{i}^{n}\right)\right)-\mathrm{E}\left(f\left(c Y_{i}^{n}\right)\right)=\frac{a}{n}\{c(f(1)-f(0))-f(c)+f(0)\} .
$$

Note that $g(1)=0$. Hence, our proof is complete if we show that $g$ is decreasing in $c>1$. Indeed,

$$
\begin{aligned}
g^{\prime}(c) & =\frac{a}{n}\left\{(f(1)-f(0))-f^{\prime}(c)\right\} \\
& =\frac{a}{n}\left\{f^{\prime}(b)-f^{\prime}(c)\right\} \\
& \leq 0, \quad b<c,
\end{aligned}
$$

where $b \in(0,1)$ by the mean value theorem and $f^{\prime}$ is increasing due to convexity.

A Poisson-Poisson cluster PP can also be dcx compared to a homogeneous Poisson PP. Let $\Phi$ and $\Phi^{\prime}$ be homogeneous Poisson PPs with intensities $\lambda<\infty$ and $\lambda \times \lambda_{0}$, respectively. Define $\mu(y)=\sum_{X_{i} \in \Phi} h\left(X_{i}-y\right)$. Let $\Phi^{\prime \prime}$ be $\operatorname{Cox}(\mu(x))$.

Proposition 5.2. Let $\Phi, \Phi^{\prime}$, and $\{\mu(y)\}$ be as above. Assume that $\mu(y)$ is a.s. locally Riemann integrable and that $\mathrm{E}(\mu(y))=\mathrm{E}(\mu(0))<\infty$. Then $\Phi^{\prime} \leq_{\mathrm{dcx}} \Phi^{\prime \prime}$.

Proof. By the last statement of Lemma 5.1 we have $\lambda(\mathrm{d} x) \leq_{\mathrm{dcx}} \Phi(\mathrm{d} x)$. Note that $\lambda \times \lambda_{0}=$ $\int_{\mathbb{R}^{d}} h(x-y) \lambda(\mathrm{d} x)$ and, thus, by the second statement of Theorem 2.1 (note the assumption $\mathrm{E}(\mu(y))<\infty),\left\{\lambda \times \lambda_{0}\right\} \leq_{\mathrm{dcx}}\{\mu(y)\}$, where the dcx smaller field is a deterministic constant. The result now follows from the second statement of Lemma A.4, by the assumption that $\mu(y)$ is a.s. Riemann integrable, and by observing that $\mathrm{E}\left(\int_{A} \mu(y) \mathrm{d} y\right)=\mathrm{E}(\mu(0)) \int_{A} \mathrm{~d} y<\infty$ for all bounded Borel subsets $A$.

Remark 5.2. Consider a Poisson PP $\Phi^{\prime}$ and $\operatorname{Cox}(\mu)$ as in Proposition 5.2. It is known that the Palm version (given a point at the origin) of $\Phi^{\prime}$ can be constructed taking $\Phi^{\prime}+\varepsilon_{0}$. By [25, Proposition 2], analogously, the Palm version of $\operatorname{Cox}(\mu)$ can be taken as $\operatorname{Cox}(\mu)+\varepsilon_{0}+\Phi^{\prime \prime}$, where $\Phi^{\prime \prime}$ is an independent $\operatorname{Cox}(\mu)$ Poisson PP with intensity $h(y-\xi)$, where $\xi$ is sampled from the distribution $h(\mathrm{~d} x) / \int h(y) \mathrm{d} y$. This shows that we cannot expect dex ordering of the Palm versions of $\Phi^{\prime}$ and $\operatorname{Cox}(\mu)$.

\subsection{Log Cox point processes}

This class of PPs is defined by the logarithm of their intensity fields.

An extension of an LCPP studied in [12] is a log Lévy-driven Cox process (LLCPP). Under the notation of the previous subsection, a PP $\Phi$ is said to be an LLCPP if its intensity field is given by

$$
\lambda(y)=\exp \left(\int_{\mathbb{R}^{d}} k(x, y) L(\mathrm{~d} x)\right) .
$$

Hellmund et al. [12] allowed for negative kernels and signed Lévy measures, but these do not fit into our framework. Suppose that $L_{1} \leq_{\text {idcx }} L_{2}$. Then $\Phi_{1} \leq_{\text {idcx }} \Phi_{2}$, where $\Phi_{i}, i=1,2$, are 
the respective LLCPPs of $L_{i}, i=1,2$, with kernel $k(\cdot, \cdot)$. These are simple consequences of Theorem 2.1 and the exponential function being icx.

Another class is the log Gaussian Cox process (LGCP) (see [27]). A PP $\Phi$ is said to be an LGCP if its intensity field is $\lambda(y)=\exp (X(y))$, where $\{X(y)\}$ is a Gaussian random field. Suppose that $\left\{X_{i}(y)\right\}, i=1,2$, are two Gaussian random fields. Then $\left\{X_{1}(y)\right\} \leq_{\text {idcx }}$ $\left\{X_{2}(y)\right\}$ if and only if $\mathrm{E}\left(X_{1}(y)\right) \leq \mathrm{E}\left(X_{2}(y)\right)$ for all $y \in \mathbb{R}^{d}$ and $\operatorname{cov}\left(X_{1}\left(y_{1}\right), X_{1}\left(y_{2}\right)\right) \leq$ $\operatorname{cov}\left(X_{2}\left(y_{1}\right), X_{2}\left(y_{2}\right)\right)$ for all $y_{1}, y_{2} \in \mathbb{R}^{d}$. From the composition rules of the idcx order, it is clear that idcx ordering of Gaussian random fields implies idcx ordering of the corresponding LGCPs. An example of a parametric dcx-ordered Gaussian random field is given in [23, Section 4].

\subsection{Generalized shot noise Cox processes (GSNCPs)}

This class of Cox PPs was first introduced, and its various statistics studied, in [26]. In simple terms, these processes are Cox PPs whose random intensity fields are shot noise fields of PPs. We say that a Cox PP is a GSNCP if the random intensity field $\{\lambda(y)\}_{y \in \mathbb{R}^{d}}$ driving the Cox PP is of the form $\lambda(y)=\sum_{j} \gamma_{j} k_{b_{j}}\left(c_{j}, y\right)$, where $\left(c_{j}, b_{j}, \gamma_{j}\right) \in \Phi$, a PP on $\mathbb{R}^{d} \times(0, \infty) \times(0, \infty)$. Also, we impose the following condition on the kernel $k: k_{b_{j}}\left(c_{j}, y\right)=k_{1}\left(c_{j} / b_{j}, y / b_{j}\right) / b_{j}^{d}$, where $k_{1}\left(c_{j}, \cdot\right)$ is a density with respect to the Lebesgue measure on $\mathbb{R}^{d}$. We shall denote the GSNCP driven by $\Phi$ as $\Phi^{\mathrm{G}}$. This class includes various known PPs, such as Neyman-Scott PPs, Thomas PPs, Matérn cluster PPs, among others. The case where the $b_{j}$ s are constants and $\left\{\left(c_{j}, \gamma_{j}\right)\right\}$ is a Poisson PP is called the shot noise Cox process (see [25]). Shot noise Cox processes are also LCPPs. Consider two PPs, $\Phi_{1}$ and $\Phi_{2}$, such that $\Phi_{1} \leq \mathrm{dcx} \Phi_{2}, \Phi_{1} \leq_{\text {idcx }} \Phi_{2}$, and $\Phi_{1} \leq_{\text {idcv }} \Phi_{2}$. Then, from Theorem 2.1, we infer that $\Phi_{1}^{\mathrm{G}} \leq_{\mathrm{dcx}} \Phi_{2}^{\mathrm{G}}, \Phi_{1}^{\mathrm{G}} \leq_{\mathrm{idcx}} \Phi_{2}^{\mathrm{G}}$, and $\Phi_{1}^{\mathrm{G}} \leq_{\mathrm{idev}} \Phi_{2}^{\mathrm{G}}$.

\subsection{Ginibre-radii-like point processes}

Let $\left\{\Phi_{i}\right\}_{i \geq 0}$ be an i.i.d. family of PPs on $\mathbb{R}^{+}$. So, the points of each PP $\Phi_{i}$ can be sequenced, based on their distance from the origin. Let $\Phi$ be the PP formed by picking the ith point of $\Phi_{i}$ for $i \geq 1$. From now on we shall abbreviate $\Phi([0, b])$ to $\Phi(b)$ for $b>0$; we similarly abbreviate the other PPs used. Note the following representations for $\Phi(b)$ and $\Phi_{0}(b)$ :

$$
\Phi(b)=\sum_{k \geq 1} 1\left[\Phi_{k}(b) \geq k\right], \quad \Phi_{0}(b)=\sum_{k \geq 1} \mathbf{1}\left[\Phi_{0}(b) \geq k\right] .
$$

Let

$$
\Phi^{m}(b)=\sum_{k \geq 1}^{m} \mathbf{1}\left[\Phi_{k}(b) \geq k\right], \quad \Phi_{0}^{m}(b)=\sum_{k \geq 1}^{m} \mathbf{1}\left[\Phi_{0}(b) \geq k\right] .
$$

By Lorentz's inequality (see [28, Theorem 3.9.8]), it follows that $\left(\Phi_{1}(b), \ldots, \Phi_{m}(b)\right) \leq_{\mathrm{sm}}$ $\left(\Phi_{0}(b), \ldots, \Phi_{0}(b)\right)$, where sm stands for supermodular (see [28, Section 3.9]). Define the function $f: \mathbb{N}^{m} \rightarrow \mathbb{R}$ as follows: $f\left(n_{1}, \ldots, n_{m}\right)=\sum_{k \geq 1} \mathbf{1}\left[n_{k} \geq k\right]$. It is easy to verify that both $f$ and $-f$ are sm and that $f(n \wedge m) \leq f(n), f(m) \leq f(n \vee m)$. Consequently, $g \circ f$ is sm, provided $g$ is cx and

$$
\begin{aligned}
\mathrm{E}\left(g\left(\Phi^{m}(b)\right)\right) & =\mathrm{E}\left(g \circ f\left(\Phi_{1}(b), \ldots, \Phi_{m}(b)\right)\right) \\
& \leq \mathrm{E}\left(g \circ f\left(\Phi_{0}(b), \ldots, \Phi_{0}(b)\right)\right) \\
& =\mathrm{E}\left(g\left(\Phi_{0}^{m}(b)\right)\right) .
\end{aligned}
$$

Hence, $\Phi^{m}(b) \leq_{\mathrm{cx}} \Phi_{0}^{m}(b)$, and using Lemma A.2, we obtain $\Phi(b) \leq_{\mathrm{cx}} \Phi_{0}(b)$. To complete the proof, that is, to prove that $\Phi \leq_{\mathrm{dcx}} \Phi_{0}$, we would require a multivariate generalization of Lorentz's inequality, which we have been unable to prove. 
We now explain the reasons for considering the above PP $\Phi$. If we assume that the $\Phi_{i}$ above are Poisson then $\Phi$ is known to be a representation of the PP of the squared radii $\left|\Phi_{\mathrm{G}}\right|^{2}=\left\{\left|X_{n}\right|^{2}: X_{n} \in \Phi_{\mathrm{G}}\right\}$ of the Ginibre process $\Phi_{\mathrm{G}}$ (see [13] and [18]). It has been observed in simulations that this determinental PP exhibits less clustering than the homogeneous Poisson PP. Our result can be seen as a first step towards a formal statement of this property.

\section{Applications to wireless communication networks}

From the point of view of applications of our main result, it remains to provide examples of interesting dex functions. In what follows, we provide such functions arising in the context of wireless networks. In many of the models we have assumed ordered PPs with i.i.d. marks. However, owing to Proposition 3.7, the results hold for independently marked Cox PPs, provided the respective intensity measures are ordered.

\subsection{Coverage process with independent grains}

The Boolean model $C(\Phi, G)$ defined earlier (see Definition 4.3) is the main object of analysis in the theory of coverage processes (see [10]). The percolation properties of the Boolean model have been studied in [22], while the connectivity properties of the Boolean model have been studied in [29]. For $\tilde{\Phi}$ as in the Definition 4.3 of the Boolean model, denote by $V(y)=\sum_{\left(X_{i}, G_{i}\right) \in \tilde{\Phi}} \mathbf{1}\left[y \in X_{i}+G_{i}\right]$ the number of grains covering $y \in \mathbb{R}^{d}$. Denote by $\psi\left(s_{1}, \ldots, s_{n}\right)$ the joint probability generating functional (PGF) of the number of grains covering locations $y_{1}, \ldots, y_{n} \in \mathbb{R}^{d}, \psi\left(s_{1}, \ldots, s_{n}\right)=\mathrm{E}\left(\prod_{j=1}^{n} s_{j}\left(y_{j}\right)\right), s_{j} \geq 0, j=1, \ldots, n$. Note that the function $g\left(v_{1}, \ldots, v_{n}\right)=\prod_{j}^{n} s_{j}^{v_{j}}$ is idcx when $s_{j} \geq 1$ for all $j=1, \ldots, n$ and is ddcx when $0 \leq s_{j} \leq 1$ for all $j$. Thus, the following result follows immediately from Theorem 2.1, Proposition 3.2, and Proposition 3.7.

Corollary 6.1. Let $\Phi_{i}, i=1,2$, be simple PPs (of germs) on $\mathbb{R}^{d}$. Consider the corresponding Boolean models with the typical grain $G$ and, as above, denote the respective coverage number fields by $\left\{V_{i}(y)\right\}$ and their $P G F$ s by $\psi_{i}$. If $\Phi_{1} \leq_{\mathrm{dcx}} \Phi_{2}, \Phi_{1} \leq_{\mathrm{idcx}} \Phi_{2}$, and $\Phi_{1} \leq_{\mathrm{idcv}} \Phi_{2}$, then $\left\{V_{1}(y)\right\} \leq_{\mathrm{dcx}}\left\{V_{2}(y)\right\},\left\{V_{1}(y)\right\} \leq_{\mathrm{idcx}}\left\{V_{2}(y)\right\}$, and $\left\{V_{1}(y)\right\} \leq_{\mathrm{idcv}}\left\{V_{2}(y)\right\}$, respectively, with the result for the dcx order holding provided that $\mathrm{E}\left(V_{i}(y)\right)<\infty$ for all $y$. In particular, if $\Phi_{1} \leq \mathrm{idcx} \Phi_{2}$ then $\mathrm{E}\left(V_{1}(y)^{\beta}\right) \leq \mathrm{E}\left(V_{2}(y)^{\beta}\right)$ for all $\beta \geq 1$. If $\Phi_{1} \leq_{\mathrm{idcx}} \Phi_{2}$ and $\Phi_{1} \leq \mathrm{ddcx}_{2} \Phi_{2}$ then $\psi_{1}\left(s_{1}, \ldots, s_{n}\right) \leq \psi_{2}\left(s_{1}, \ldots, s_{n}\right)$ for $s_{j} \geq 1$ and, respectively, $s_{j} \leq 1, j=1, \ldots, n$.

Note that $1-\psi(0, \ldots, 0)$ represents the expected coverage measure, i.e. the probability of whether locations $y_{1}, \ldots, y_{n}$ are covered by at least one grain. It was shown in [10, Section 3.8] that the expected one-point coverage (or volume fraction in the case of stationary PPs) for a stationary Cox PP and some clustered PPs is lower than that of a stationary, homogeneous Poisson PP.

Coverage processes arise in various applications. In particular, in wireless communications the points of the PP (germs) usually represent locations of antennas and their grains the respective communication regions. In this context, $V(y)$ is the number of antennas covering the point $y$ and the coverage measure is the indicator that at least one of them is able to reach $y$. The application of the Boolean model to the modeling of wireless communications dates back to Gilbert [8] in 1961.

\subsection{Random geometric graphs (RGGs)}

This class of graphs has increasingly found applications in spatial networks. For a detailed study of these graphs, see [29]. A random geometric graph is defined as a graph with vertex 
set $\Phi$ and edge set $E=\left\{\left\{X_{i}, X_{j}\right\}:\left|X_{i}-X_{j}\right| \leq r\right\}$. Clearly, this is related to the Boolean model defined in the previous subsection. One of the objects of interest in an RGG is the typical degree. Under the notation of the previous subsection, the typical degree (denoted by $\operatorname{deg}(\Phi, G)$ ) for an RGG formed by a stationary PP $\Phi$ and grain distribution $G$ is

$$
\operatorname{deg}(\Phi, G)=\frac{1}{\lambda|A|} \sum_{X_{i}, X_{j} \in \Phi} \mathbf{1}\left[X_{i} \in A\right] \mathbf{1}\left[X_{i} \neq X_{j}\right] \mathbf{1}\left[\left(X_{i}+G_{i}\right) \cap\left(X_{j}+G_{j}\right) \neq \varnothing\right],
$$

where $A$ is a bounded Borel subset. If $G=B_{0}(r), r>0$, then $\mathrm{E}(\operatorname{deg}(\Phi, G))=K(r)$ is Ripley's $K$ function defined in Section 3.2. Corollary 6.2, below, follows easily from Theorem 2.1, Proposition 3.3, and Proposition 3.7.

Corollary 6.2. Consider two simple PPs, $\Phi_{1}$ and $\Phi_{2}$, such that $\Phi_{1} \leq_{\mathrm{dcx}} \Phi_{2}$. Then

$$
\operatorname{deg}\left(\Phi_{1}, G\right) \leq_{\mathrm{idcx}} \operatorname{deg}\left(\Phi_{2}, G\right) .
$$

\subsection{Interference in wireless communications}

The Boolean model is not sufficient for analyzing wireless networks, as it ignores the fact that in radio communications signals received from one particular transmitter are jammed by the signals received from the other transmitters. According to information theory, as well as existing technology, the quality of a given radio communication link is determined by the so-called signal-to-interference-and-noise ratio (SINR) at the receiver of this link. From a mathematical point of view, the interference in the above considerations is just the sum of the powers of the signals received from all transmitters (except perhaps own transmitter(s)). It is then the shot noise field of received powers that plays an important role in determining the connectivity and the capacity of the network in a broad sense. The foundations of the theory of SINR coverage processes are quite recent (see [1], [2], [6], and [9]). In what follows, we study the impact of the structure of the PPs of interferers on given radio links.

Consider a set of $n$ emitters $\left\{x_{i}\right\}$ and $n$ receivers $\left\{y_{i}\right\}$. Suppose that the signal received by $y_{i}$ from $x_{k}$ is $S_{k i}$. These $\left\{S_{i k}\right\}$ are assumed to be independent. The assumption of independence is due to the phenomenon of fading. Let the set of additional interferers be modeled by an i.i.d. marked PP $\tilde{\Phi}=\varepsilon_{\left(X_{j},\left(Z_{j}^{1}, \ldots, Z_{j}^{n}\right)\right)}$, independent of $\left\{S_{i k}\right\}$, where $Z_{j}^{n}$ is the power received by the receiver $y_{i}$ from the interferer located at $X_{j}$. Denote the background noise RV by $W$.

We say that the signal from $x_{i}$ is successfully received by $y_{i}$ if $S_{i i} /\left(W+I_{i}+V_{i}\right)>T$, where $I_{i}=\sum_{k \neq i} S_{k i}$ is the interference received at $y_{i}$ from the set of other emitters $\left\{x_{k}: k \neq i\right\}$, the $V_{i}=\sum_{j} Z_{j}^{i}$ are the interferers in $\tilde{\Phi}$, and $T>0$ is some required SINR threshold (assumed to be constant). Let $p$ denote the probability of successful reception of signals from each $x_{i}$ to $y_{i}$. Then

$$
p=\mathrm{P}\left(S_{i i}>\left(W+I_{i}+V_{i}\right) T \text { for all } i=1, \ldots, n\right)=\mathrm{E}\left(\prod_{i} \bar{F}_{i i}\left(T\left(W+I_{i}+V_{i}\right)\right)\right)
$$

where $\bar{F}_{i i}(s)=\mathrm{P}\left(S_{i i} \geq s\right)$ and the second equality is due to independence. Given $\left\{I_{i}: i=\right.$ $1, \ldots, n\}$ and $W$, the expression under the expectation in (6.1) can be viewed as a function of the value of the shot noise vector $\left(V_{1}, \ldots, V_{n}\right)$ evaluated with respect to $\tilde{\Phi}$. Theorem 2.1 and Proposition 3.7 imply the following result concerning the impact of the structure of the set of interferers on $p$. 
Corollary 6.3. Consider emitters $\left\{x_{i}\right\}$, receivers $\{y\}_{i}$, and powers $\left\{S_{k i}\right\}$ as above. Let $\tilde{\Phi}_{u}, u=$ 1,2, be two simple marked PPs of interferers. Denote by $p_{u}, u=1,2$, the probability of successful reception given by (6.1) in the model with the set of interferers $\tilde{\Phi}_{u}$. Assume that the product of the tail distribution functions of the received powers, $\prod_{i=1}^{n} \bar{F}_{i i}\left(s_{i}\right)$, are dcx. If $\Phi_{1} \leq \mathrm{ddcx} \Phi_{2}$ then $p_{1} \leq p_{2}$.

It is quite natural to assume that $\prod_{i=1}^{n} \bar{F}_{i i}\left(s_{i}\right)$ is ddcx. For example, the constant emitted power $P$, omni-directional path-loss function $l(r)$, and Rayleigh fading in the radio channel implies that $S_{k i}=P H_{k i} / l\left(\left|x_{k}-y_{i}\right|\right)$, where $\left|H_{k i}\right|$ are i.i.d. exponential RVs with mean 1 . In this case, $\prod_{i=1}^{n} \bar{F}_{i i}\left(s_{i}\right)$ is ddcx. Recently, in [7], under the assumption of Rayleigh fading, direct analytical methods have been used to compare the probability of successful reception in Poisson PPs and a class of Poisson-Poisson cluster PPs, known as Neyman-Scott PPs, for both stationary and Palm versions. These results rely on explicit expressions for this probability to be known in the considered cases. Furthermore, it is shown that, for a certain choice of parameters, the Palm version of the Poisson-Poisson cluster PP has a worse probability of successful reception than the Poisson PP. In our terminology, this simply means that the corresponding Palm versions are not ddcx-ordered, as the connectivity probability is a ddcx function (6.1) of the integral shot noise fields of the corresponding Palm versions. This strengthens Remark 5.2 by showing that idcx ordering of Palm versions is the best we can obtain in full generality.

\section{Conclusions and open questions}

To the best of the authors' knowledge, this is the first study of dcx ordering of random measures and PPs. We have defined the dcx order and characterized it using finite-dimensional distributions of the measure values on disjoint, bounded Borel subsets of the space. In our main result we proved that the integrals of some nonnegative kernels with respect to dcx-ordered random measures inherit this ordering from the measures. This was shown to be a very useful tool in the study of many particular characteristics of random measures, and in the construction and analysis of stochastic models.

In this paper we have left several open questions. Here we briefly summarize them.

- Our dex order is defined via finite-dimensional distributions of random measures. This makes the verification of the dcx order easier, but requires additional work when studying functionals, which cannot be explicitly expressed in terms of the values of the measure on some finite collection of bounded Borel subsets as, for example, an integral of the measure. Considering a dcx ${ }^{1 *}$ order on the space of measures could facilitate the former task. However, the precise regularity conditions of the $\mathrm{dcx}^{1 *}$ functional on the space of measures which would guarantee the equivalence between these two approaches are not known (cf. Section 3.5).

- Comparisons of Ripley's functions (see Proposition 3.4) and pair correlation functions (Corollary 3.1) seem to indicate that PPs higher in the dcx order cluster more. We have shown examples of PPs which are larger than Poisson PPs, namely Cox PPs, which indeed exhibit more clustering than Poisson PPs. It would be interesting to show examples of PPs which are dcx smaller than Poisson PPs, and which exhibit less clustering. Matérn 'hard core' PPs and Ginibre PPs are some natural candidates for this.

- We have studied dcx orders that take into account the dependence structure and the variability of the marginals or random measures. It seems plausible to study in a similar manner other orders such as the convex order, the componentwise convex order, etc. Note, 
however, that the supermodular order does not seem to be a reasonable one in the context of random measures. The reason being that it allows us to compare only measures with the same finite-dimensional distributions, and, thus, a Poisson PP can only be (trivially) compared in this order to itself. Indeed, Poisson finite-dimensional distributions imply the total independence property and, thus, uniquely characterize Poisson PPs (cf. [5, Lemma 2.3.I]).

\section{Appendix A}

In order to make the paper more self-contained, we now recall some basic results on stochastic orders used in the main stream of the paper.

Lemma A.1. ([28, Chapter 3].) 1. A twice differentiable function $f$ is directionally convex if and only if for all $x$,

$$
\frac{\partial^{2}}{\partial x_{i} \partial x_{j}} f(x) \geq 0, \quad 1 \leq i, j \leq n .
$$

2. The stochastic order relation ' $\leq_{\mathrm{dcx}}$ ' is generated by infinitely differentiable dcx functions.

Owing to the above lemma, in some places we only prove that two random vectors are ordered with respect to twice differentiable dcx functions and conclude that they are dcx-ordered.

We denote by ' $\stackrel{\mathrm{D}}{\rightarrow}$ ' convergence in distribution (weak convergence).

Lemma A.2. ([28, Chapter 3].) Let $\left(X^{(k)}: k=1, \ldots\right)$ and $\left(Y^{(k)}: k=1, \ldots\right)$ be sequences of random vectors. Suppose that $X^{(k)} \leq_{\mathrm{dcx}} Y^{(k)}$ for all $k \in \mathbb{N}$. If $X^{(k)} \stackrel{\mathrm{D}}{\rightarrow} X$ and $Y^{(k)} \stackrel{\mathrm{D}}{\rightarrow} Y$, and if, moreover, $\mathrm{E}\left(X^{(k)}\right) \rightarrow \mathrm{E}(X)$ and $\mathrm{E}\left(Y^{(k)}\right) \rightarrow \mathrm{E}(Y)$, then $X \leq_{\mathrm{dcx}} Y$.

Lemma A.3. ([20, Lemmas 2.17 and 2.18].) 1. For $i=1, \ldots, m$, let $\left(S_{j}^{i}: j=1, \ldots\right)$ be independent sequences of i.i.d. nonnegative RVs. If $f$ is $d c x$, idcx, or idcv, then $g\left(n_{1}, \ldots, n_{m}\right)=$ $\mathrm{E}\left(f\left(\sum_{j=1}^{n_{1}} S_{j}^{1}, \ldots, \sum_{j=1}^{n_{m}} S_{j}^{m}\right)\right)$ is also dcx, idcx, or idcv, respectively.

2. Let $N_{i}, i=1, \ldots, k$, denote $k$ mutually independent Poisson $R V s$, where the mean of $N_{i}$ is $\lambda_{i}$. If $\phi: \mathbb{N}^{k} \rightarrow \mathbb{R}$ is $d c x$, idcx, or idcv, then $g\left(\lambda_{1}, \ldots, \lambda_{k}\right)=\mathrm{E}\left(\phi\left(N_{1}, \ldots, N_{k}\right)\right)$ is also dcx, $i d c x$, or idcv, respectively.

The first part of the following lemma is an easy extension of the one-dimensional version in [20]. The second part, which we prove in what follows, is a further extension of it.

Lemma A.4. Suppose that $\{X(s)\}_{s \in \mathbb{R}^{d}}$ and $\{Y(s)\}_{s \in \mathbb{R}^{d}}$ are two nonnegative, real-valued, and a.s. locally Riemann integrable random fields. For some $n \geq 1$ and disjoint, bounded Borel subsets $I_{1}, \ldots, I_{n}$, define $J_{X}^{i}=\int_{I_{i}} X(s) \mathrm{d} s$ and $J_{Y}^{i}=\int_{I_{i}} Y(s) \mathrm{d} s$.

1. If $\{X(s)\} \leq_{\mathrm{idcx}}\{Y(s)\}$ or $\{X(s)\} \leq_{\mathrm{idcv}}\{Y(s)\}$, then $\left(J_{X}^{1}, \ldots, J_{X}^{n}\right) \leq_{\mathrm{idcx}}\left(J_{Y}^{1}, \ldots, J_{Y}^{n}\right)$ or $\left(J_{X}^{1}, \ldots, J_{X}^{n}\right) \leq_{\mathrm{idcv}}\left(J_{Y}^{1}, \ldots, J_{Y}^{n}\right)$, respectively, for any $n$ and any disjoint, bounded Borel subsets $I_{1}, \ldots, I_{n}$.

2. Suppose further that $\mathrm{E}\left(\int_{A} X(x) \mathrm{d} x\right)<\infty$ for all bounded Borel subsets $A$ in $\mathbb{R}^{d}$, and similarly for $\{Y(x)\}$. If $\{X(x)\} \leq_{\mathrm{dcx}}\{Y(x)\}$ then $\left(J_{X}^{1}, \ldots, J_{X}^{n}\right) \leq_{\mathrm{dcx}}\left(J_{Y}^{1}, \ldots, J_{Y}^{n}\right)$.

Proof. We prove part 2 for $d=1$, and, as can be seen from the proof, the generalization is fairly straightforward.

We need to prove that $\left(\int_{I_{1}} X(s) \mathrm{d} s, \ldots, \int_{I_{n}} X(s) \mathrm{d} s\right) \leq_{\mathrm{dcx}}\left(\int_{I_{1}} Y(s) \mathrm{d} s, \ldots, \int_{I_{n}} Y(s) \mathrm{d} s\right)$ for $I_{i}, i=1, \ldots, n$, disjoint bounded Borel subsets. We give an approximation satisfying the 
assumptions of Lemma A.2. Let $I_{i}=\left[a_{i}, b_{i}\right], a_{i}, b_{i} \in \mathbb{R}, i=1, \ldots, n$. Let $\left\{\left(t_{m j}^{i}\right)_{1 \leq j \leq k_{m}}, i=\right.$ $1, \ldots, n\}$ be the sequences of the $m$ th nested partition of each interval. The middle Riemann sum can be given as $X^{m}\left(I_{i}\right)=\sum_{j} X\left(t_{m j}^{i}\right)\left(t_{m(j+1)}^{i}-t_{m j}^{i}\right), i=1, \ldots, n, k \in \mathbb{N}$, and similarly for $Y(x)$. These are the variables satisfying the approximation in Lemma A.2. As $X(s)$ is Riemann integrable,

$$
\left(X^{m}\left(I_{1}\right), \ldots, X^{m}\left(I_{n}\right)\right) \rightarrow\left(J_{X}^{1}, \ldots, J_{X}^{n}\right) \text { a.s. }
$$

and, hence, in distribution. It is also clear that the middle Riemann sums of $X(\cdot)$ and $Y(\cdot)$ are ordered. What remains to prove is that $\mathrm{E} X^{m}\left(I_{i}\right) \rightarrow \mathrm{E} J_{X}^{i}$. In the last term, by Fubini, we can interchange the expectation and integral and, hence, it suffices to prove that $\mathrm{E}\left(X^{m}\left(I_{i}\right)\right) \rightarrow$ $\int_{I_{i}} \mathrm{E}(X(s)) \mathrm{d} s$. Our assumption implies that this is true.

\section{Acknowledgements}

DY was supported by a grant from EADS, France. This work has greatly benefitted from discussions with Professor Francois Baccelli. DY also wishes to thank Professor Fred Huffer for sharing his technical report.

\section{References}

[1] Baccelli, F. ANd BŁaszczyszyn, B. (2001). On a coverage process ranging from the Boolean model to the Poisson-Voronoi tessellation with applications to wireless communications. Adv. Appl. Prob. 33, 293-323.

[2] Baccelli, F., BŁaszczyszyn, B. And MüHlethaler, P. (2006). An aloha protocol for multihop mobile wireless networks. IEEE Trans. Inf. Theory 52, 421-436.

[3] Bassan, B. And Scarsini, M. (1991). Convex orderings for stochastic processes. Comment. Math. Univ. Carolin. 32, 115-118.

[4] Chang, C.-S., Chao, X. and Pinedo, M. (1991). Monotonicity results for queues with doubly stochastic Poisson arrivals: Ross's conjecture. Adv. Appl. Prob. 23, 210-228.

[5] Daley, D. J. And Vere-Jones, D. (1988). An Introduction to the Theory of Point Processes. Springer, New York.

[6] Dousse, O. et al. (2006). Percolation in the signal to interference ratio graph. J. Appl. Prob. 43, 552-562.

[7] Ganti, R. And HaengGi, M. (2008). Interference and outage in clustered wireless ad hoc networks. Preprint. Available at http://arxiv.org/abs/0706.2434

[8] Gilbert, E. N. (1961). Random plane networks. J. SIAM 9, 533-543.

[9] Gupta, P. and Kumar, P. R. (2000). The capacity of wireless networks. IEEE Trans. Inf. Theory 42, 388-404.

[10] Hall, P. G. (1988). Introduction to the Theory of Coverage Processes. John Wiley, New York.

[11] Heinrich, L. ANd Molchanov, I. S. (1994). Some limit theorems for extremal and union shot-noise processes. Math. Nachr. 168, 139-159.

[12] Hellmund, G., Prokešová, M. and Vedel Jensen, E. B. (2008). Lévy-based Cox point processes. Adv. Appl. Prob. 40, 603-629.

[13] Hough, J. B., Krishnapur, M., Peres, Y. and Virag, B. (2006). Determinantal processes and independence. Prob. Surveys 3, 206-229.

[14] Huffer, F. (1984). Inequalities for $M / G / \infty$ queues and related shot noise processes. Tech. Rep. 351, Department of Statistics, Stanford University.

[15] Huffer, F. (1987). Inequalities for the $M / G / \infty$ queue and related shot noise processes. J. Appl. Prob. 24, 978-989.

[16] Kallenberg, O. (1983). Random Measures. Academic Press, London.

[17] Kamae, T., Krengel, U. and O'Brien, G. L. (1977). Stochastic inequalities on partially ordered spaces. Ann. Prob. 5, 899-912.

[18] Kostlan, E. (1992). On the spectra of Gaussian matrices. Linear Algebra Appl. 162/164, 385-388.

[19] Lindvall, T. (1992). Lectures on the Coupling Method. John Wiley, London.

[20] Meester, L. E. and Shanthikumar, J. G. (1993). Regularity of stochastic processes: a theory based on directional convexity. Prob. Eng. Inf. Sci. 7, 343-360.

[21] Meester, L. E. and Shanthikumar, J. G. (1999). Stochastic convexity on general space. Math. Operat. Res. 24, 472-494.

[22] Meester, R. and Roy, R. (1996). Continuum Percolation. Cambridge University Press. 
[23] Miyoshi, N. (2004). A note on bounds and monotonicity of spatial stationary Cox shot noise. Prob. Eng Inf. Sci. 18, 561-571.

[24] MiYoshi, N. ANd Rolski, T. (2004). Ross-type conjectures on monotonicity of queues. Austral. N. Z. J. Statist. 46, 121-131.

[25] Møller, J. (2003). Shot noise Cox processes. Adv. Appl. Prob. 35, 614-640.

[26] Møller, J. And Torrisi, G. L. (2005). Generalized shot noise Cox processes. Adv. Appl. Prob. 37, 48-74.

[27] Møller, J., Syversveen, A. R. and WaAgepetersen, R. P. (1998). Log Gaussian Cox processes. Scand. J. Statist. 25, 451-482.

[28] Müller, A. And Stoyan, D. (2002). Comparison Methods for Stochastic Models and Risks. John Wiley, New York.

[29] Penrose, M. D. (2003). Random Geometric Graphs. Oxford University Press.

[30] Rolski, T. (1986). Upper bounds for single server queues with doubly stochastic Poisson arrivals. Math. Operat. Res. 11, 442-450.

[31] Rolski, T. ANd Szekli, R. (1991). Stochastic ordering and thinning of point processes. Stoch. Process. Appl. 37, 299-312.

[32] Ross, S. M. (1978). Average delay in queues with non-stationary Poisson arrivals. J. Appl. Prob. 15, $602-609$.

[33] Shaked, M. and Shanthikumar, J. G. (1990). Parametric stochastic convexity and concavity of stochastic processes. Ann. Inst. Statist. Math. 42, 509-531.

[34] Stoyan, D. (1983). Inequalities and bounds for variances of point processes and fibre processes. Math. Operationsforsch. Statist. Ser. Statist. 14, 409-419.

[35] Stoyan, D., Kendall, W. S. And Mecke, J. (1995). Stochastic Geometry and Its Applications. John Wiley, Chichester. 